\title{
Extended one-dimensional method for coherent synchrotron radiation including shielding
}

\author{
David Sagan, Georg Hoffstaetter, Christopher Mayes, and Udom Sae-Ueng \\ Cornell University, Ithaca, New York 14853, USA \\ (Received 17 June 2008; published 28 April 2009)
}

\begin{abstract}
Coherent synchrotron radiation can severely limit the performance of accelerators designed for high brightness and short bunch length. Examples include light sources based on energy recovery LINAC or free-electron lasers, and bunch compressors for linear colliders. In order to better simulate coherent synchrotron radiation, a one-dimensional formalism due to Saldin, Schneidmiller, and Yurkov has been implemented in the general beam dynamics code Bmad. Wide vacuum chambers are simulated by means of vertical image charges. Results from Bmad are here compared to analytical approximations, to numerical solutions of the Maxwell equations, and to the simulation code ELEGANT and the code of Agoh and Yokoya.
\end{abstract}

DOI: 10.1103/PhysRevSTAB.12.040703

PACS numbers: 41.60.Ap, 41.20.Jb, 29.27.Bd

\section{INTRODUCTION}

It is envisioned that future accelerators will call for shorter beams of higher intensity. A possible limiting factor in these efforts is an increase in energy spread and transverse emittance, as well as a microbunching instability, due to coherent synchrotron radiation (CSR).

The first CSR calculations were performed by Schwinger in 1945. Using a Green's function method, he arrives at the power spectrum of a single charge bending in free space as well as between infinite conducting plates, and thereby computes the coherent power radiated by a collection of charges [1]. Warnock extends this work to include the longitudinal impedance on a bunched beam [2]. Many papers covering the history and importance of CSR forces can be found in [3].

This paper uses an approach to calculate the CSR wakefield originating with Saldin, Schneidmiller, and Yurkov [4] which is a generalization of a prior calculation [5,6]. The calculation starts with the CSR force between two charges traveling on the same curve, and integrates over a longitudinal bunch distribution to give a longitudinal wakefield. Transverse particle coordinates and transverse force components are neglected. The formalism can be applied to any arbitrary coplanar lattice configuration of bends and drifts, including, for example, radiation from one bend entering another.

Simulating CSR effects is the subject of a number of codes. The method here is implemented in the particle tracking code Bmad [7]. Our simulation results are compared with approximate analytic formulas as well as with two of the codes described by Bassi [8] - the simulation code ELEGANT and the code of Agoh and Yokoya.

\section{TWO PARTICLE INTERACTION}

The CSR analysis starts by considering two particles of charge $e$ following the same trajectory as shown in Fig. 1 .
The Liénard-Wiechert formula [9] gives the electric field $\mathbf{E}(\mathbf{P})$ at the position of the kicked particle at point $\mathbf{P}$ and time $t$ due to the source particle at point $\mathbf{P}^{\prime}$ and retarded time $t^{\prime}$ :

$$
\begin{aligned}
\mathbf{E}(\mathbf{P})= & \frac{e}{4 \pi \epsilon_{0}} \\
& \times \frac{\frac{1}{\gamma^{2}}\left(\mathbf{L}-L \beta \mathbf{n}^{\prime}\right)+\frac{1}{c^{2}} \mathbf{L} \times\left[\left(\mathbf{L}-L \beta \mathbf{n}^{\prime}\right) \times \mathbf{a}^{\prime}\right]}{\left(L-\mathbf{L} \cdot \beta \mathbf{n}^{\prime}\right)^{3}} .
\end{aligned}
$$

It will be assumed that both particles have the same constant speed $\beta=v / c$. The unit velocity vectors for the source and kicked particles are $\mathbf{n}^{\prime}$ and $\mathbf{n}$, respectively, The retarded time $t^{\prime}$ is related to $t$ via $t-t^{\prime}=L / c$, where $\mathbf{L}=\mathbf{P}-\mathbf{P}^{\prime}$ is the vector from the source point to the kick point. At time $t$, the source particle has a longitudinal position $z^{\prime}$ with respect to the bunch center and the longitudinal position of the kicked particle is $z$. The distance $\zeta \equiv z-z^{\prime}$ between the particles at constant time can be computed via the equation

$$
\zeta=L_{s}-\beta L,
$$

where $L_{s}$ is the path length from $\mathbf{P}^{\prime}$ to $\mathbf{P}$. Generally, the

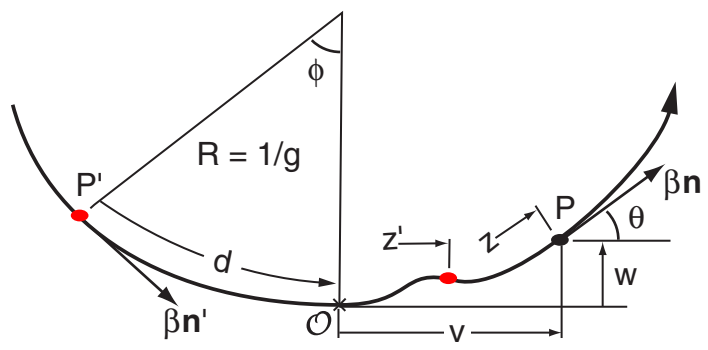

FIG. 1. (Color) The radiation from a particle at point $\mathbf{P}^{\prime}$ kicks a particle at point $\mathbf{P}$. 
relativistic approximation $\beta=1$ will be made. However, some terms in $1-\beta \simeq 1 / 2 \gamma^{2}$ will need to be retained.

The first term on the right-hand side of Eq. (1) has a $1 / \zeta^{2}$ singularity at small distances. Following Saldin et al. [6], this singularity is dealt with by dividing the electric field into two parts. The space charge component $\mathbf{E}_{\mathrm{SC}}$, which contains the singularity, is the field that would result if the particles where moving without acceleration along a straight line. The CSR term, $\mathbf{E}_{\mathrm{CSR}}$, is what is left after subtracting off the space charge term

$$
\mathbf{E}_{\mathrm{SC}} \equiv \frac{e}{4 \pi \epsilon_{0}} \frac{\operatorname{sign}(\zeta) \mathbf{n}}{\gamma^{2} \zeta^{2}}, \quad \mathbf{E}_{\mathrm{CSR}} \equiv \mathbf{E}-\mathbf{E}_{\mathrm{SC}}
$$

where $\operatorname{sign}(\zeta)$ is 1 for positive and -1 for negative $\zeta$. The rate $K \equiv d \mathcal{E} / d s$ at which the kicked particle is changing energy due to the field of the source particle is

$$
K \equiv K_{\mathrm{CSR}}+K_{\mathrm{SC}}=e \mathbf{n} \cdot \mathbf{E}_{\mathrm{CSR}}+e \mathbf{n} \cdot \mathbf{E}_{\mathrm{SC}}
$$

Following Saldin et al. [6], the transverse extent of the beam will be ignored in the calculation of $K_{\mathrm{CSR}}$. However, the inclusion of the finite beam size will be needed to remove the singularity in the calculation of $K_{\mathrm{SC}}$ as discussed in Sec. IV.

\section{CSR CALCULATION}

The source point $\mathbf{P}^{\prime}$ and the kick point $\mathbf{P}$ will, in general, not be within the same lattice element. Because the transverse extent of the beam is being ignored, all elements will be considered to be either bends or drifts and it will be assumed that the geometry is coplanar.

In Fig. $1, R$ is the bending radius and $g=1 / R$ is the bending strength of the element that contains the source point $\mathbf{P}^{\prime}$. The magnitude of the acceleration is $a^{\prime} \simeq c^{2} / R$. This element ends at point $\mathcal{O}$. The angle between $\mathbf{P}^{\prime}$ and $\mathcal{O}$ is $\phi$, and $d=R \phi$ is the path length between $\mathbf{P}^{\prime}$ and $\mathcal{O}$.

Between point $\mathcal{O}$ and the kick point $\mathbf{P}, d_{i}$ is the path length within the $i$ th element, $i=1, \ldots, N$, where $N$ is the number of elements in this region. For the last element, $d_{N}$ is the distance from the start of the element to point $\mathbf{P}$. For the $i$ th element, $\phi_{i}$ is the bend angle, $R_{i}$ is the bend radius, and $g_{i}=1 / R_{i}$ is the bend strength. For a drift $\phi_{i}=$ $g_{i}=0$.

In Fig. $1,(v, w)$ are the coordinates of point $\mathbf{P}$ with respect to point $\mathcal{O}$ with the $v$ axis parallel to the orbit's longitudinal $s$ axis at point $\mathcal{O}$, and the $w$ axis pointing upwards towards the inside of the element containing the point $\mathbf{P}^{\prime}$.

With this notation, the difference in $v$ and $w$ from the beginning of an element to the end is

$$
\begin{aligned}
\Delta v_{i} & = \begin{cases}R_{i}\left(\sin \left(\phi_{i}+\psi_{i}\right)-\sin \psi_{i}\right) & \text { for a bend } \\
d_{i} \cos \psi_{i} & \text { for a drift }\end{cases} \\
\Delta w_{i} & = \begin{cases}R_{i}\left(\cos \psi_{i}-\cos \left(\phi_{i}+\psi_{i}\right)\right) & \text { for a bend } \\
d_{i} \sin \psi_{i} & \text { for a drift, }\end{cases}
\end{aligned}
$$

where $\psi_{i}$ is the orientation angle at the entrance end of the element

$$
\psi_{i}=\sum_{k=1}^{i-1} \phi_{k}
$$

The above formulas are able to handle negative bends (beam rotating clockwise). For a negative bend $R_{i}, g_{i}$ and $\phi_{i}$ are negative while $d_{i}=R_{i} \phi_{i}$ is always positive.

With the assumption that all bend angles are small, $v$ and $w$ can be approximated by

$$
v=\nu_{1}-\nu_{3}, \quad \text { and } \quad w=\omega_{2},
$$

where

$$
\begin{aligned}
& \nu_{1}=\sum_{i=1}^{N} d_{i}, \quad \omega_{2}=\sum_{i=1}^{N} d_{i}\left(\psi_{i}+\frac{1}{2} g_{i} d_{i}\right), \\
& \nu_{3}=\sum_{i=1}^{N} d_{i}\left(\frac{1}{2} \psi_{i}^{2}+\frac{1}{2} \psi_{i} g_{i} d_{i}+\frac{1}{6} g_{i}^{2} d_{i}^{2}\right),
\end{aligned}
$$

and the small angles have been retained to second order.

The angle $\theta$ of the vector $\mathbf{n}$ with respect to the $v$ axis is $\theta=\sum_{i=1}^{N} g_{i} d_{i}$. In the $(v, w)$ coordinate system, the components of the vector $\mathbf{L}$ are

$$
\begin{aligned}
L_{v} & =v+R \sin \phi=\left[\nu_{1}+d\right]-\left[\nu_{3}+\frac{g^{2} d^{3}}{6}\right], \\
L_{w} & =w-R(1-\cos \phi)=\omega_{2}-\frac{g d^{2}}{2}, \\
L & =\sqrt{L_{v}^{2}+L_{w}^{2}} \\
& =\left[\nu_{1}+d\right]-\left[\nu_{3}+\frac{g^{2} d^{3}}{6}-\frac{1}{8} \frac{\left(2 \omega_{2}-g d^{2}\right)^{2}}{\nu_{1}+d}\right] .
\end{aligned}
$$

The path length is simply

$$
L_{s}=d+\sum_{i=1}^{N} d_{i}=d+\nu_{1} .
$$

This, with Eq. (2), gives

$$
\zeta=\frac{\nu_{1}+d}{2 \gamma^{2}}+\left[\nu_{3}+\frac{g^{2} d^{3}}{6}-\frac{1}{8} \frac{\left(2 \omega_{2}-g d^{2}\right)^{2}}{\nu_{1}+d}\right]
$$

where terms to second order in combinations of angles and $1 / \gamma$ are retained. Substituting these expressions into Eq. (1), and defining

$$
\begin{gathered}
\alpha=\gamma^{2}\left(\omega_{2}+g d \nu_{1}+\frac{1}{2} g d^{2}\right), \quad \kappa=\gamma(\theta+g d), \\
\tau=\gamma\left(d+\nu_{1}\right),
\end{gathered}
$$

the individual terms in Eq. (1) read as 


$$
\begin{gathered}
\frac{1}{\left(L-\mathbf{L} \cdot \beta \mathbf{n}^{\prime}\right)^{3}}=\frac{8 \gamma^{9} \tau^{3}}{\left(\tau^{2}+\alpha^{2}\right)^{3}}, \\
\mathbf{n} \cdot\left(\mathbf{L}-L \beta \mathbf{n}^{\prime}\right) / \gamma^{2}=\frac{\tau^{2}-\alpha^{2}+2 \tau \alpha \kappa}{2 \gamma^{5} \tau}, \\
\mathbf{n} \cdot\left(\mathbf{L} \times\left[\left(\mathbf{L}-L \beta \mathbf{n}^{\prime}\right) \times \mathbf{a}^{\prime}\right]\right) / c^{2}=\frac{g\left(\tau^{2}-\alpha^{2}\right)(\alpha-\tau \kappa)}{2 \gamma^{5} \tau} .
\end{gathered}
$$

Putting these together yields

$$
\begin{aligned}
K_{\mathrm{CSR}}= & 4 r_{c} m c^{2} \gamma^{4} \tau^{2}\left\{\frac{g\left(\tau^{2}-\alpha^{2}\right)(\alpha-\tau \kappa)}{\left(\tau^{2}+\alpha^{2}\right)^{3}}\right. \\
& \left.+\frac{\tau^{2}-\alpha^{2}+2 \tau \alpha \kappa}{\left(\tau^{2}+\alpha^{2}\right)^{3}}\right\}-\frac{r_{c} m c^{2}}{\gamma^{2} \zeta^{2}} .
\end{aligned}
$$

While we have used SI units, the classical radius $r_{c}$ and the mass $m$ have been used to make the formula independent of the unit system.

From Eq. (14), $K_{\mathrm{CSR}}^{\circ}$, which is $K_{\mathrm{CSR}}$ restricted to the special case where points $\mathbf{P}$ and $\mathbf{P}^{\prime}$ are within the same bend, reduces to Eq. (32) of Saldin et al. [6],

$$
\begin{aligned}
K_{\mathrm{CSR}}^{\circ}= & \frac{4 r_{c} m c^{2} \gamma^{4}}{R^{2}}\left\{\frac{\hat{\phi}^{2} / 4-1}{2\left(1+\hat{\phi}^{2} / 4\right)^{3}}\right. \\
& \left.+\frac{1}{\hat{\phi}^{2}}\left[\frac{1+3 \hat{\phi}^{2} / 4}{\left(1+\hat{\phi}^{2} / 4\right)^{3}}-\frac{1}{\left(1+\hat{\phi}^{2} / 12\right)^{2}}\right]\right\},
\end{aligned}
$$

where $\hat{\phi} \equiv \gamma \phi, \alpha=R \hat{\phi}^{2} / 2, \kappa=\hat{\phi}$, and $\tau=R \hat{\phi}$. This equation is valid for $\hat{\phi}>0$; for $\hat{\phi}<0, K_{\mathrm{CSR}}$ is, to a very good approximation, zero.

In the limit of small $\zeta, K_{\mathrm{CSR}}^{\circ}$ has a limiting value of

$$
K_{\mathrm{CSR}}^{\circ}(\zeta) \simeq \frac{-4 r_{c} m c^{2} \gamma^{4}}{3 R^{2}} \quad \text { for } \zeta \ll \frac{R}{\gamma^{3}}
$$

At large values of $\zeta, \zeta$ is cubic in $\phi$ so that $\phi \simeq$ $(24 \zeta / R)^{1 / 3}$. With this, Eq. (15) becomes

$$
K_{\mathrm{CSR}}^{\circ}(\zeta) \simeq \frac{2 r_{c} m c^{2}}{3^{4 / 3} R^{2 / 3} \zeta^{4 / 3}} \quad \text { for } \zeta \gg \frac{R}{\gamma^{3}},
$$

which corresponds to Eq. (10) of Saldin et al. [6] [note the error in the denominator of Saldin et al. Eq. (10)]. Figure 2 shows $K_{\mathrm{CSR}}^{\circ}(\zeta)$, which changes sign at $\zeta \approx 1.8 R / \gamma^{3}$. The long tail at $\zeta>1.8 R / \gamma^{3}$ cannot be neglected since the integral $\int_{0}^{\infty} d \zeta K_{\mathrm{CSR}}(\zeta)$ is zero. The vanishing of the integral is a reflection of the fact that a closed loop of charged particles of uniform density does not radiate.

The fact that $K_{\mathrm{CSR}}$ is highly peaked in amplitude near $\zeta=0$ can be problematic for simulations at ultrarelativistic energies because the characteristic longitudinal distance between particles or mesh points needs to be less than

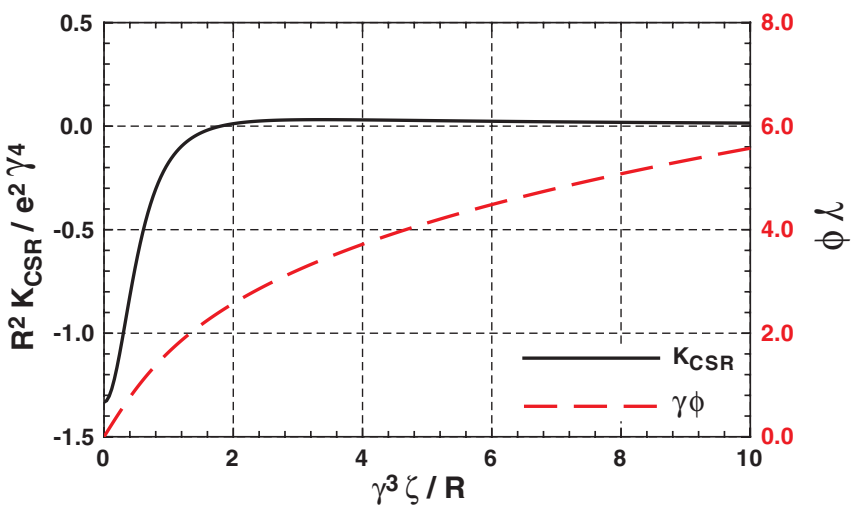

FIG. 2. (Color) $K_{\mathrm{CSR}}^{\circ}$ (left) and $\phi$ (right) as a function of $\zeta$ for a bend.

$R / \gamma^{3}$. One way of dealing with the peaked nature of $K_{\mathrm{CSR}}$ is to first consider the kick from a line of particles of density $\lambda(z)$ and then to integrate by parts

$$
\begin{aligned}
\left(\frac{d \mathcal{E}}{d s}\right)_{\mathrm{CSR}} & =\int_{-\infty}^{\infty} d z^{\prime} \lambda\left(z^{\prime}\right) K_{\mathrm{CSR}}\left(z-z^{\prime}\right) \\
& =\int_{-\infty}^{\infty} d z^{\prime} \frac{d \lambda\left(z^{\prime}\right)}{d z^{\prime}} I_{\mathrm{CSR}}\left(z-z^{\prime}\right),
\end{aligned}
$$

where

$$
I_{\mathrm{CSR}}\left(z-z^{\prime}\right)=-\int_{-\infty}^{z^{\prime}} d z^{\prime \prime} K_{\mathrm{CSR}}\left(z-z^{\prime \prime}\right)
$$

$I_{\mathrm{CSR}}^{\circ}$, which is $I_{\mathrm{CSR}}$ for $\mathbf{P}$ and $\mathbf{P}^{\prime}$ in the same bend, is plotted in Fig. 3. The peaked nature of $K_{\mathrm{CSR}}$ has been smoothed over at the cost of having to deal with a derivative of $\lambda$. For $\zeta \gg R / \gamma^{3}$, the approximation of Eq. (17) can be used to calculate an explicit ultrarelativistic equation for $I_{\mathrm{CSR}}^{\circ}$ as in [10],

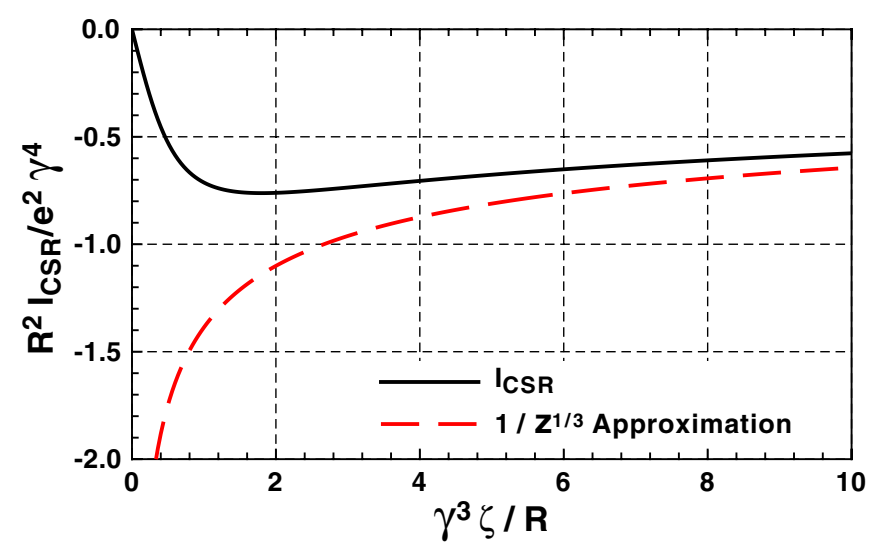

FIG. 3. (Color) $I_{\mathrm{CSR}}^{\circ}$ as a function of $\zeta$ for a bend. The dashed line is the large $\zeta$ approximation as given in Eq. (21). 


$$
I_{\mathrm{CSR}}^{\circ}(\zeta)=\frac{-2 r_{c} m c^{2}}{3^{1 / 3} R^{2 / 3}} \frac{1}{\zeta^{1 / 3}} \quad \text { for } \zeta \gg \frac{R}{\gamma^{3}} .
$$

Equation (21) is also plotted in Fig. 3.

While, in general, it is helpful to have explicit formulas, for the purposes of evaluation within a simulation program this is not needed. The alternative is to use an exact implicit solution. Because Eqs. (11) and (14) are rational functions, Eq. (20) can be integrated. The last term that compensates $K_{\mathrm{SC}}$ can be integrated to $r_{c} m c^{2} /\left(\gamma^{2} \zeta\right)$. The other terms can be written with

$$
\frac{\partial \zeta}{\partial d}=\frac{\tau^{2}+\alpha^{2}}{2 \gamma^{2} \tau^{2}}
$$

as

$$
\begin{aligned}
K_{\mathrm{CSR}}= & 2 r_{c} m c^{2} \gamma^{2}\left(\frac{\partial \zeta}{\partial d}\right)^{-1}\left\{\frac{g\left(\tau^{2}-\alpha^{2}\right)(\alpha-\tau \kappa)}{\left(\tau^{2}+\alpha^{2}\right)^{2}}\right. \\
& \left.+\frac{\tau^{2}-\alpha^{2}+2 \tau \alpha \kappa}{\left(\tau^{2}+\alpha^{2}\right)^{2}}\right\}+\frac{\partial}{\partial \zeta}\left(\frac{r_{c} m c^{2}}{\gamma^{2} \zeta}\right) .
\end{aligned}
$$

With $\partial \tau / \partial d=\gamma, \partial \alpha / \partial d=\gamma g \tau$, and $\partial \kappa / \partial d=\gamma g$, one can further simplify to

$$
K_{\mathrm{CSR}}=-2 r_{c} m c^{2} \gamma\left(\frac{\partial \zeta}{\partial d}\right)^{-1} \frac{\partial}{\partial d}\left(\frac{\tau+\alpha \kappa}{\tau^{2}+\alpha^{2}}\right)+\frac{\partial}{\partial \zeta}\left(\frac{r_{c} m c^{2}}{\gamma^{2} \zeta}\right)
$$

This can be integrated over $\zeta$ to yield

$$
I_{\mathrm{CSR}}\left(z, z^{\prime}\right)=-r_{c} m c^{2}\left(\frac{2 \gamma(\tau+\alpha \kappa)}{\tau^{2}+\alpha^{2}}-\frac{1}{\gamma^{2} \zeta}\right) .
$$

It can be shown that, while quantities like $d$ and $g$ are discontinuous across element boundaries, $\tau, \alpha$, and $\kappa$ are continuous and, hence, $I_{\mathrm{CSR}}$ is a continuous function as it should be.

Equation (25), to second order, is equivalent to Eq. (10) of Ref. [4]. Using Eq. (25), the integration of Eq. (19) in a simulation program can be done via interpolation of Eq. (11). Equation (25) has several advantages over equations like Eq. (21). It is applicable at lower values of $\gamma^{3} \zeta$, that is, at lower energies and/or smaller length scales. Additionally, Eq. (25) has no singularity at small $\zeta$, and it can be used to handle any combination of elements between the source and kick points.

\section{SPACE CHARGE CALCULATION}

The singularity at small $\zeta$ in the space charge field $\mathbf{E}_{\mathrm{SC}}$ in Eq. (3) is removed by considering the finite transverse beam size. This term is equivalent to the problem of calculating the field given a static distribution of charges. It will be assumed that at any longitudinal position the transverse profile of the beam is Gaussian. Thus, a longitudinal slice of the beam will produce an energy change for a particle at longitudinal $z$ and transverse offset $(x, y)$ from the slice center of

$$
\begin{aligned}
d K_{\mathrm{SC}}\left(x, y, z ; z^{\prime}\right)= & \int_{-\infty}^{\infty} \int_{-\infty}^{\infty} d x^{\prime} d y^{\prime} \rho\left(x^{\prime}, y^{\prime}, z^{\prime}\right) d z^{\prime} \\
& \times \frac{r_{c} m c^{2} \gamma \zeta}{\left[\gamma^{2} \zeta^{2}+\left(x-x^{\prime}\right)^{2}+\left(y-y^{\prime}\right)^{2}\right]^{3 / 2}},
\end{aligned}
$$

where $\zeta \equiv z-z^{\prime}$ and $\rho$ is the bi-Gaussian distribution

$$
\rho(x, y, z)=\frac{\rho(z)}{2 \pi \sigma_{x} \sigma_{y}} \exp \left[-\frac{x^{2}}{2 \sigma_{x}^{2}}-\frac{y^{2}}{2 \sigma_{y}^{2}}\right] .
$$
law

In the limit $\zeta \rightarrow \pm 0, d K_{\mathrm{SC}}$ can be obtained from Gauss's

$$
d K_{\mathrm{SC}}(\zeta \rightarrow \pm 0)=\frac{r_{c} m c^{2} \operatorname{sign}(\zeta) \rho\left(z^{\prime}\right) d z^{\prime}}{\sigma_{x} \sigma_{y} \exp \left[\frac{x^{2}}{2 \sigma_{x}^{2}}+\frac{y^{2}}{2 \sigma_{y}^{2}}\right]} .
$$

For $x=y=0$ and small $\zeta$ the space charge kick to first order is

$$
d K_{\mathrm{SC}}(x=y=0)=\frac{r_{c} m c^{2} \operatorname{sign}(\zeta) \rho\left(z^{\prime}\right) d z^{\prime}}{\sigma_{x} \sigma_{y}+\frac{\pi}{2} \frac{\sigma_{x}^{2}+\sigma_{y}^{2}}{\sigma_{x}+\sigma_{y}} \gamma|\zeta|}+o\left(\zeta^{2}\right) .
$$

In the limit of $\zeta \rightarrow \infty$, the beam slice can be treated as a point particle and

$$
d K_{\mathrm{SC}}(\zeta \rightarrow \infty)=\frac{r_{c} m c^{2} \operatorname{sign}(\zeta) \rho\left(z^{\prime}\right) d z^{\prime}}{\gamma^{2} \zeta^{2}}
$$

In general, Eq. (26) cannot be solved in closed form but a reasonable approximation for space charge calculations can be obtained by combining the above three limiting cases using a quadratic in the denominator:

$$
d K_{\mathrm{SC}} \approx \frac{r_{c} m c^{2} \operatorname{sign}(\zeta) \rho\left(z^{\prime}\right) d z^{\prime}}{\sigma_{x} \sigma_{y} \exp \left[\frac{x^{2}}{2 \sigma_{x}^{2}}+\frac{y^{2}}{2 \sigma_{y}^{2}}\right]+\frac{\sigma_{x}^{2}+\sigma_{y}^{2}}{\sigma_{x}+\sigma_{y}} \gamma|\zeta|+\gamma^{2} \zeta^{2}} .
$$

The factor of $\pi / 2$ in the linear term in Eq. (29) is dropped in Eq. (31) since this gives a slightly better fit overall. While Eq. (31) is not accurate for large transverse displacements $\left(|x|>3 \sigma_{x}\right.$ or $\left.|y|>3 \sigma_{y}\right)$, both the actual and approximate $d K_{\mathrm{SC}}$ are small in this region compared with the kick for particles near $x=y=0$ so this inaccuracy will have a negligible effect on any simulations.

That Eq. (31) is a reasonable approximation is illustrated in Figs. 4 and 5 which show $K_{\mathrm{SC}}$ as a function of $z$ as computed from an integration of Eq. (26) and from the approximate Eq. (31) for a round beam and for a beam with $\sigma_{y} / \sigma_{x}=1 / 100$ aspect ratio. The particular parameters 


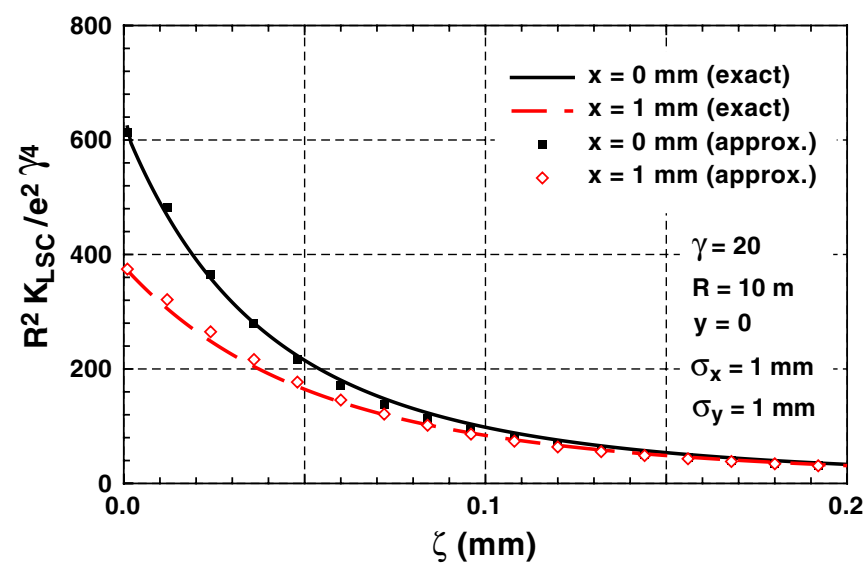

FIG. 4. (Color) Comparison between the approximation of Eq. (31) and an exact integration of Eq. (26) for a round beam.

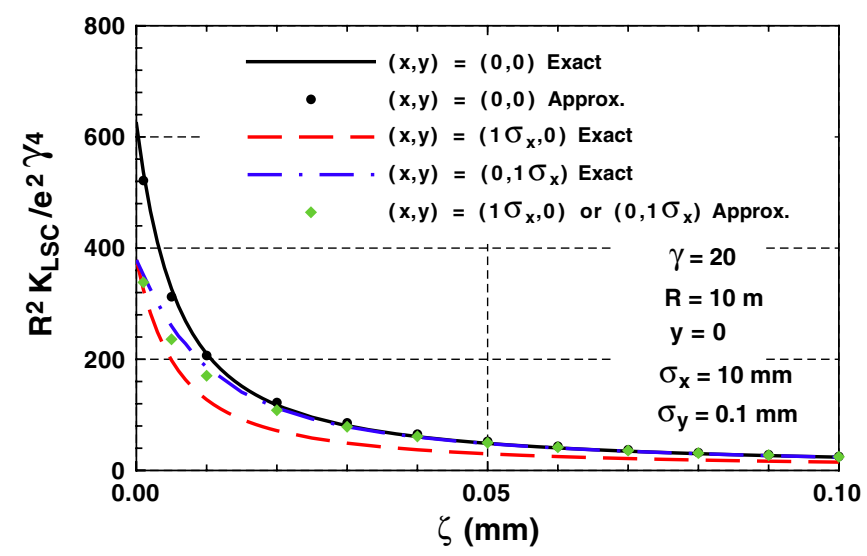

FIG. 5. (Color) Comparison between the approximation of Eq. (31) and an exact integration of Eq. (26) for a beam with $\sigma_{y} / \sigma_{x}=0.01$

chosen for the computation are given in the figure. Two cases were considered. One where the kicked particle is on axis, and the other where the kicked particle is displaced by $x=\sigma_{x}$ or $y=\sigma_{y}$ off axis.

At high energies, the CSR energy kick is independent of the beam energy as indicated by Eq. (21). On the other hand, the factors of $\gamma$ in the denominator of Eq. (31) ensure the $K_{\mathrm{SC}}$ will be a decreasing function of $\gamma$. Above some energy, the effect of $K_{\mathrm{SC}}$ will be small compared to the effect of $K_{\mathrm{CSR}}$. To estimate that point, consider the maximum energy kick in a Gaussian bunch of $N_{e}$ particles with

$$
\rho(z)=\frac{N_{e}}{\sqrt{2 \pi} \sigma_{z}} \exp \left[-\frac{z^{2}}{2 \sigma_{z}^{2}}\right]
$$

The space charge kick is maximum at $x, y=0$ and using Eq. (31) gives

$$
\begin{aligned}
K_{\mathrm{SC}}(0,0, z) \approx & \frac{N_{e} r_{c} m c^{2}}{\sqrt{2 \pi} \sigma_{z}} \\
& \times \int_{0}^{\infty} d \zeta \frac{\exp \left[-\frac{(z-\zeta)^{2}}{2 \sigma_{z}^{2}}\right]-\exp \left[-\frac{(z+\zeta)^{2}}{2 \sigma_{z}^{2}}\right]}{\sigma_{x} \sigma_{y}+\frac{\sigma_{x}^{2}+\sigma_{y}^{2}}{\sigma_{x}+\sigma_{y}} \gamma \zeta+\gamma^{2} \zeta^{2}}
\end{aligned}
$$

The dominant term in the denominator in the integrand is either $\sigma_{x} \sigma_{y}$ for small $\zeta$, or $\gamma^{2} \zeta^{2}$ for large $\zeta$. As an approximation, the middle term in the denominator will therefore be ignored. The approximation $\sinh \left(z \zeta / \sigma_{z}^{2}\right) \approx$ $z \zeta / \sigma_{z}^{2}$ will also be made. This approximation is justified since, as seen below, the region of maximum $K_{\mathrm{SC}}$ is around $z \approx \sigma_{z}$ and for realistic beam parameters, $\sigma_{x} \sigma_{y} \ll \gamma^{2} \sigma_{z}^{2}$. Therefore, the only significant contribution to the integral will come in the region $\zeta<\sigma_{z}$. With these approximations, Eq. (33) becomes

$$
K_{\mathrm{SC}}(z) \approx \frac{2 N_{e} r_{c} m c^{2} z \exp \left[-\frac{z^{2}}{2 \sigma_{z}^{2}}\right]}{\sqrt{2 \pi} \sigma_{z}^{3}} \int_{0}^{\infty} d \zeta \frac{\zeta \exp \left[-\frac{\zeta^{2}}{2 \sigma_{z}^{2}}\right]}{\sigma_{x} \sigma_{y}+\gamma^{2} \zeta^{2}}
$$

The integral can be evaluated to $\left(2 \gamma^{2}\right)^{-1} e^{a} \operatorname{Ei}(a)$, where $\mathrm{Ei}$ is the exponential integral and $a=\sigma_{x} \sigma_{z} /\left(2 \gamma^{2} \sigma_{z}^{2}\right)$. For small $a$, this is approximately $-\ln (a)$. The maximum of $K_{\mathrm{SC}}$ occurs at $z=\sigma_{z}$. Using $\sigma_{x} \sigma_{y} \ll \gamma^{2} \sigma_{z}^{2}$ then gives

$$
\begin{aligned}
K_{\mathrm{SC}}, \max & \approx-\frac{\exp \left[-\frac{1}{2}\right]}{\sqrt{2 \pi}} \frac{N_{e} r_{c} m c^{2}}{\sigma_{z}^{2} \gamma^{2}} \log \left(\frac{\sigma_{x} \sigma_{y}}{2 \gamma^{2} \sigma_{z}^{2}}\right) \\
& \approx-\frac{N_{e} r_{c} m c^{2}}{4 \sigma_{z}^{2} \gamma^{2}} \log \left(\frac{\sigma_{x} \sigma_{y}}{2 \gamma^{2} \sigma_{z}^{2}}\right) .
\end{aligned}
$$

On the other hand, The maximum CSR kick at large energies is, from Eq. (14),

$$
K_{\mathrm{CSR}}, \max \approx 0.8 \frac{N_{e} r_{c} m c^{2}}{\left(R^{2} \sigma_{z}^{4}\right)^{1 / 3}} .
$$

The ratio is

$$
\frac{K_{\mathrm{SC}}, \max }{K_{\mathrm{CSR}}, \max } \approx\left[0.3 \log \left(\frac{2 \gamma^{2} \sigma_{z}^{2}}{\sigma_{x} \sigma_{y}}\right)\right] \frac{1}{\gamma^{2}}\left(\frac{R}{\sigma_{z}}\right)^{2 / 3} .
$$

$K_{\mathrm{SC}} / K_{\mathrm{CSR}}$ will be small when

$$
\gamma \gg M\left(\frac{R}{\sigma_{z}}\right)^{1 / 3}
$$

where

$$
M=\left[0.3 \log \left(\frac{2 \gamma^{2} \sigma_{z}^{2}}{\sigma_{x} \sigma_{y}}\right)\right]^{1 / 2}
$$


$M$ is slowly varying and, for many practical problems, of order unity. In this case, $\sigma_{z}$ must be interpreted as the characteristic longitudinal distance over which the bunch density is changing.

Condition Eq. (38) is similar to the condition for the validity of the ultrarelativistic Eq. (21). From Fig. 21, The condition for validity of Eq. (21) is seen to be

$$
\gamma \ll\left(\frac{R}{\zeta}\right)^{1 / 3} .
$$

\section{CSR IN BMAD}

The above algorithm for simulating CSR and longitudinal space charge (SC) has been implemented as part of the Bmad [7] subroutine library for relativistic chargedparticle simulations. Bmad simulates a beam as a set of particles. To simulate CSR, the beam is tracked through a lattice element by dividing the element into a number of slices. Tracking through a slice involves first propagating the particles independently from each other and then applying the CSR and SC energy kicks. To calculate the energy kick, the beam is divided longitudinally into $N_{b}$ bins as shown in Fig. 6. For the purposes of computing the charge in each bin, each beam particle is considered to have a triangular charge distribution. The overlap of the triangular charge distribution with a bin determines that particle's contribution to the total charge in the bin. The width of the particle's triangular charge distribution and the number of bins are set by the user. The bin width is adjusted at each time step so that the bins will span the bunch length. Increasing the particle width smooths the distribution at the cost of resolution.

The charge density $\lambda_{i}$ at the center of the $i$ th bin is taken to be $\lambda_{i}=\rho_{i} / \Delta z_{b}$, where $\rho_{i}$ is the total charge within the bin and $\Delta z_{b}$ is the bin width. The charge density is assumed to vary linearly in between the bin centers. The CSR energy kick for a particle at the center of the $j$ th bin after traveling a distance $d s_{\text {slice }}$ according to Eq. (19) is then

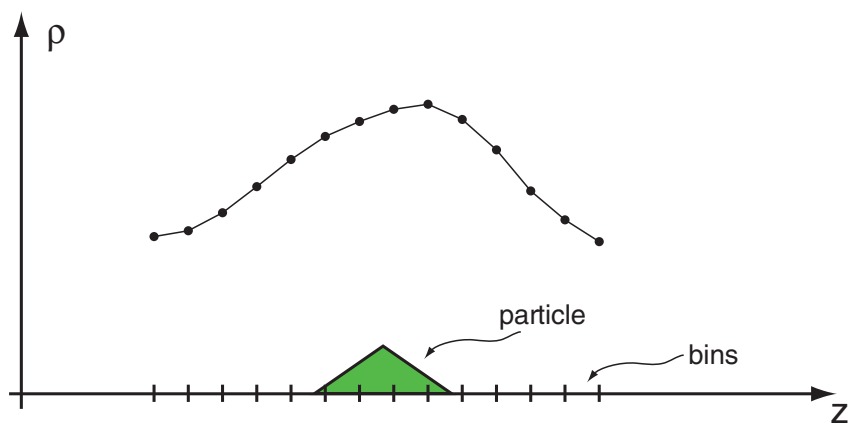

FIG. 6. (Color) Bmad implementation of the CSR algorithm. The beam of particles is divided up into a number of bins. The contribution of a particle to a bin's total charge is determined by the overlap of the particle's triangular charge distribution and the bin.

$$
\begin{aligned}
d \mathcal{E}_{j}= & d s_{\text {slice }} \sum_{i=1}^{N_{b}}\left(\lambda_{i}-\lambda_{i-1}\right) \\
& \times \frac{I_{\mathrm{CSR}}(j-i)+I_{\mathrm{CSR}}(j-i+1)}{2},
\end{aligned}
$$

where

$$
I_{\mathrm{CSR}}(j) \equiv I_{\mathrm{CSR}}\left(z=j \Delta z_{b}\right) .
$$

Evaluation of $I_{\mathrm{CSR}}(j)$ involves inversion of Eq. (11) to obtain $d$. Because $z$ is a monotonic function of $d$, Newton's method [11] is used to find numbers $d_{1}$ and $d_{2}$ which bracket the root and then Ridders' method [11] is used to quickly find $d$.

In deriving Eq. (41), the approximation

$$
\int_{j \Delta z_{b}}^{(j+1) \Delta z_{b}} d z I_{\mathrm{CSR}}(z) \approx \Delta z_{b} \frac{I_{\mathrm{CSR}}(j)+I_{\mathrm{CSR}}(j+1)}{2}
$$

has been used. Generally this is an excellent approximation, except when $j=0$ and $\Delta z_{b} \gg R / \gamma^{3}$, as shown in Fig. 3. Here, however, the integral can be done exactly assuming that the source and kick points lie within the same element:

$$
\int_{0}^{\Delta z_{b}} d z I_{\mathrm{CSR}}(z)=\frac{1}{\gamma^{2}} \ln \left(\frac{2 \gamma^{2} \Delta z_{b}}{d\left(\Delta z_{b}\right)}\right)-\frac{d\left(\Delta z_{b}\right)^{2} g^{2}}{4} .
$$

Once the energy kick at the centers of the bins is calculated, the energy kick applied to a particle is calculated via interpolation assuming a linear variation of the kick between bin centers.

In calculating the energy kick, the computational time for calculating the charge in the bins scales as $N_{p}$, the number of particles in the simulation. The computational time for calculating the energy kick at the bin centers scales as $N_{b}^{2}$, and the time for calculating the energy kick of the particles scales as $N_{p}$.

\section{A. Chamber walls}

The Bmad simulation incorporates the shielding of the top and bottom chamber walls by using image currents. The Appendix explains why neglecting the width of a chamber is a good approximation when the width is larger than the height. Since the image currents are well separated from the actual beam, there are no singularities to deal with, and a direct integration of the kick without subtracting off $K_{\mathrm{SC}}$ is possible. Using Eqs. (14) and (18), the image current kick is calculated in Bmad via the equation

$$
\begin{aligned}
d \mathcal{E}_{j}(\text { image })= & 2 * d s_{\text {slice }} \sum_{k=1}^{N_{i}}(-1)^{k} \\
& \times \sum_{i=1}^{N_{b}} q_{i} * K\left(z=(j-i) \Delta z_{b}, y=k h\right),
\end{aligned}
$$


where $q_{i}=\lambda_{i} \Delta z_{b}$ is the charge in a bin, $h$ is the chamber height, and $k$ indexes the image currents at vertical displacement $y= \pm k h$. The number of image charges $N_{i}$ needs to be chosen large enough so that the neglected image currents do not have a significant effect on the simulation results. Because the relevant angles are not small, the image charge kick $K$ must be calculated without the small angle approximation, as in Eq. (14).

\section{AGOH AND YOKOYA CSR CALCULATION}

Agoh and Yokoya (A\&Y) have developed a code to calculate CSR wakefields by directly integrating Maxwell's equations on a mesh representing a rectangular beam chamber [12]. The approach depends on the paraxial approximation, a rigid Gaussian bunch density, small chamber dimensions relative to the bending radius, and ultrarelativistic particles. Using a comoving coordinate system $(x, y, s)$ in Fourier space, they are able to reduce the problem to a tractable two-dimensional differential equation,

$$
\frac{\partial}{\partial s} \mathbf{E}_{\perp}=\frac{i}{2 k}\left[\left(\nabla_{\perp}^{2}+\frac{2 k^{2} x}{R}\right) \mathbf{E}_{\perp}-\frac{1}{\epsilon_{0}} \nabla_{\perp} \rho_{0}\right],
$$

where $\mathbf{E}_{\perp}$ is a complex two-dimensional vector related to the perpendicular electric field, $\rho_{0}$ is the charge density, $k$ is the wave number, and $R$ is the magnet bending radius. It is solved using a finite-differencing method.

\section{CSR IN ELEGANT}

The particle tracking code ELEGANT (version 17.2.2) uses Eq. (47) to compute CSR kicks without shielding by a vacuum chamber [13]. The charge distribution $\lambda(z)$ and its derivative $d \lambda / d z$ are calculated by binning the macroparticles and then employing a smoothing filter.

\section{CSR WAKE FORMULA}

\section{A. Transient effects at magnet edges}

Using retarded fields, Saldin et al. [6] derive, in the ultrarelativistic limit, a formula for the wakefield due to a bunch entering from a drift region into a bend

$$
\begin{aligned}
\left(\frac{d \mathcal{E}}{d s}\right)= & -\frac{2 N_{e} r_{c} m c^{2}}{3^{1 / 3} R^{2 / 3}}\left\{\frac{\lambda\left(s-s_{L}\right)-\lambda\left(s-4 s_{L}\right)}{s_{L}^{1 / 3}}\right. \\
& \left.+\int_{s-s_{L}}^{s} \frac{1}{\left(s-s^{\prime}\right)^{1 / 3}} \frac{d \lambda}{d s^{\prime}} d s^{\prime}\right\}, \\
s_{L} \equiv & \frac{R \phi^{3}}{24},
\end{aligned}
$$

where $\phi$ is the angle traveled into the magnet by the bunch center. Equation (47) reduces to the free space steady-state wakefield of Eq. (21) in the limit $s_{L} \rightarrow \infty$.

As worked out by Emma and Stupakov [10], synchrotron radiation will continue to propagate and affect the bunch beyond the end of a bending magnet. For a finite magnet of length $L_{m}$, an ultrarelativistic bunch at a distance $x$ from the end of this magnet experiences the free space exit wakefield

$$
\begin{gathered}
\left(\frac{d \mathcal{E}}{d s}\right)_{\mathrm{exit}}=N_{e} r_{c} m c^{2}\left\{4 \frac{\lambda\left[s-\Delta s\left(L_{m}\right)\right]}{L_{m}+2 x}\right. \\
\left.-\int_{0}^{L_{m}} \frac{4}{l+2 x} \lambda^{\prime}[s-\Delta s(l)] \frac{\partial \Delta s(l)}{\partial l} d l\right\}, \\
\Delta s(l) \equiv \frac{l^{3}}{24 R^{2}} \frac{l+4 x}{l+x}
\end{gathered}
$$

\section{B. Steady-state CSR in a bend}

CSR effects in a vacuum chamber have been computed by the Green's function of grounded parallel plates [1,2]. These formulas are difficult to compute numerically, due to the presence of high order Bessel functions, so we will use an excellent approximation developed by A\&Y [12]. The impedance for the steady state in a dipole with horizontal plates separated by a distance $h$ is

$$
\begin{gathered}
Z(k)=Z_{0} \frac{2 \pi}{h}\left(\frac{2}{k R}\right)^{1 / 3} \sum_{p=0}^{\infty} F_{\mathrm{AY}}\left(\beta_{p}^{2}\right), \\
F_{\mathrm{AY}}(x) \equiv \operatorname{Ai}^{\prime}(x)\left[\operatorname{Ai}^{\prime}(x)-i \operatorname{Bi}^{\prime}(x)\right] \\
+x \operatorname{Ai}(x)[\operatorname{Ai}(x)-i \operatorname{Bi}(x)], \\
\beta_{p} \equiv(2 p+1) \frac{\pi}{h}\left(\frac{R}{2 k^{2}}\right)^{1 / 3},
\end{gathered}
$$

where $Z_{0}=c \mu_{0}$ is the free space impedance, $k$ is the wave number, and $\mathrm{Ai}$ and $\mathrm{Bi}$ are Airy functions. The parallel plate wakefield due to a bunch with longitudinal density $\lambda(z)$ is obtained by Fourier transform:

$$
\begin{gathered}
\left(\frac{d \mathcal{E}}{d s}\right)_{\mathrm{pp}}=-N_{e} r_{c} m c^{2} \Re\left(\frac{4}{Z_{0}} \int_{0}^{\infty} Z(k) \tilde{\lambda}(k) e^{i k s} d k\right), \\
\tilde{\lambda}(k)=\int_{-\infty}^{\infty} \lambda(z) e^{-i k z} d z .
\end{gathered}
$$

\section{COMPARISON BETWEEN BMAD, AGOH AND YOKOYA, AND ELEGANT}

In order to validate our method, we compare simulations from Bmad to those using the A\&Y code and ELEGANT. For ease of reading, all magnet and bunch parameters used are enumerated in Table I, and will be referred to by the corresponding letter. Additionally, all wakefields in the graphs are normalized by

$$
W_{0}=\frac{N_{e} r_{c} m c^{2}}{\left(R^{2} \sigma_{z}^{4}\right)^{1 / 3}},
$$


TABLE I. Parameters used in simulations.

\begin{tabular}{lcccccr}
\hline \hline Set & $\begin{array}{c}R \\
(\mathrm{~m})\end{array}$ & $\begin{array}{c}L \\
(\mathrm{~m})\end{array}$ & $\begin{array}{c}w \\
(\mathrm{~cm})\end{array}$ & $\begin{array}{c}h \\
(\mathrm{~cm})\end{array}$ & $\begin{array}{c}\sigma_{z} \\
(\mathrm{~mm})\end{array}$ & $\begin{array}{c}W_{0} \\
(\mathrm{keV} / \mathrm{m})\end{array}$ \\
\hline A & 10.0 & 3.0 & 50.0 & 2.0 & 0.3 & 96.4 \\
B & 10.0 & 1.0 & 34.0 & 28.0 & 0.3 & 96.4 \\
C & 10.0 & 1.0 & 10.0 & 10.0 & 0.3 & 96.4 \\
D & 10.0 & 1.0 & 60.0 & 10.0 & 0.3 & 96.4 \\
E & 1.20 & 0.419 & 0.762 & 0.3 & 0.036 & 6695.5 \\
F & 2.22 & 0.678 & 1.71 & 2.54 & 1.0 & 52.8 \\
G & 87.9 & 6.574 & 8.0 & 4.0 & 0.3 & 22.6 \\
\hline \hline
\end{tabular}

which approximately describes the maximum amplitude of the CSR wake $d \mathcal{E} / d s$.

All simulations used a bunch charge of $1 \mathrm{nC}$, and an energy of $1 \mathrm{GeV}$. For Bmad, the nominal simulation parameters were $N_{b}=800$ bins, $N_{p}=4 \times 10^{5}$ macroparticles, $N_{i}=32$ image charges, a tracking step of $d s_{\text {step }}=0.1 \mathrm{~m}$, and a triangular bin width, of 16 bins. Variations in the initial distribution due to statistical noise and the finite number of particles can have a profound effect on the results. This is illustrated in Fig. 7 which, using parameter set $\mathrm{A}$, shows energy loss as a function of longitudinal position for four different cases. The four plots of Fig. 7 show the effect of varying the number particles, the number of bins, as well as the algorithm for generating the initial distribution. The top left plot, which used 10000 particles, had its initial distribution generated using the pseudorandom number generator RAN from Numerical Recipes [11]. This is to be compared to the top right plot which is the same as the top left except the "quasirandom" number generator SOBSEQ [11] is used in place of RAN. This generator generates a distribution that is more uniform than the uncorrelated points of the pseudorandom generator and virtually eliminates the statistical noise of the top left plot. The statistical noise can also be reduced, at the cost of computation time, by increasing the number of particles as shown in the bottom left. In this case the number of particles is 100000 but the noise is still greater than the case using the quasirandom generator. The bottom right plot is similar to the bottom left plot except the number of bins was decreased to 200 and the particle width was decreased to 8 bins thus keeping the true particle width constant. As can be seen, this does not materially affect the results; however, the computation time and the resolution are reduced by reducing the number of bins.

\section{A. Steady-state case}

Figure 8 shows the steady-state CSR kick in a bend as a function of $z$ for various values of the chamber height. The parameters used correspond to set A of Table I and are the same as used for Fig. 1 of A\&Y [12].
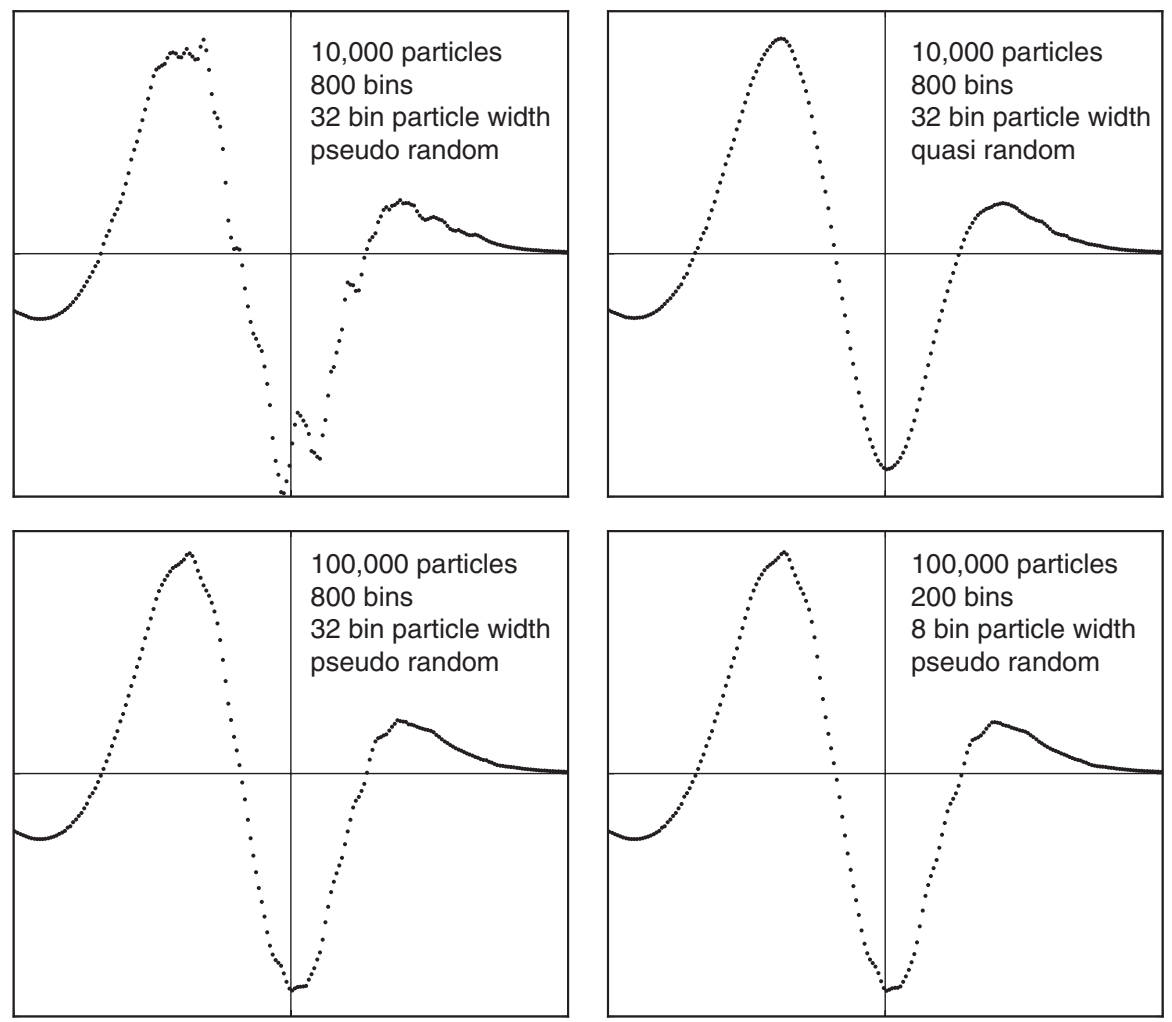

FIG. 7. Energy loss versus longitudinal position from a Bmad simulation using parameter set A. The four graphs differ in the number of particle, number of bins, particle width, and the generator used to initialize the particles. 


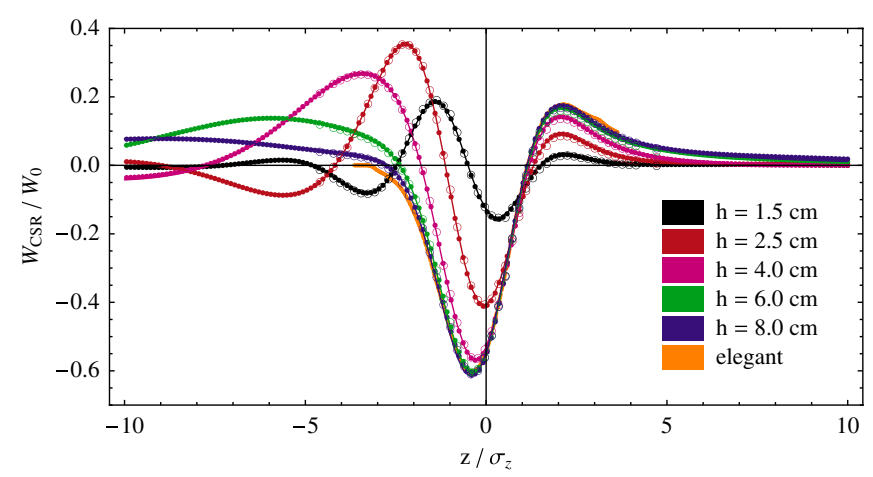

FIG. 8. (Color) The steady state using parameter set A for varying chamber heights $h$. The A\&Y code (dots), Bmad (circles), and the CSR-wake formula Eq. (53) (lines) agree well, and ELEGANT agrees with the data at large chamber heights.

Figure 8 shows excellent agreement among the A\&Y code, Bmad, and the CSR-wake formula Eq. (53).

\section{B. Transient case}

Parameter sets $\mathrm{B}, \mathrm{C}$, and $\mathrm{D}$ are used to explore the transient case where some of the kick is generated from particles in the drift region before the bend. Set B corresponds to values used in Fig. 3 of Agoh and Yokoya [12], which has a chamber of relatively large cross section. Figure 9 (left) shows agreement between Bmad, the A\&Y code, and the CSR-wake formula Eq. (47). However, for parameter set C in Fig. 9 (right), where the chamber is smaller, as in Fig. 4 of Agoh and Yokoya, there are some differences to be noted for $z / \sigma_{z}>2$. The difference between Eq. (47) and Agoh and Yokoya was noted by them and is explained by the effect of the finite chamber size which is not included in Eq. (47). In contrast, Bmad, which includes the effect of the top and bottom walls but not the side walls, is in good agreement with A\&Y, even though the chamber height and chamber width are equal.
Parameter set $\mathrm{D}$, which is the same as set $\mathrm{C}$ except that here the chamber width is much larger than the height, is used to analyze whether the chamber width is responsible for this difference. Figure 10 shows that the difference, which is larger for longer magnets, remains and thus is due to the reduced chamber height. Varying the number of Fourier coefficients used in the A\&Y calculation in Fig. 11 does not change this result, verifying that we chose a reasonable number of Fourier coefficients for solving the Maxwell equations numerically. Reversing the set $\mathrm{D}$ height and width, as in Fig. 12, somewhat reduces the difference. This indicates that the effect is due to the reduced chamber height. Practically speaking, the difference in these wakefields only becomes appreciable far in front of the bunch, where there are few particles to affect. How strong the deviation becomes for especially small chamber heights is displayed in Fig. 13 which, again using parameter sets B and $\mathrm{C}$, and using a chamber height of $h=2 \mathrm{~cm}$, shows the CSR wake as a function of $z$. Again, Bmad and A\&Y are in fairly good agreement.

\section{Realistic magnets}

To evaluate how significant the differences are in realistic magnets, we use parameter sets $\mathrm{E}, \mathrm{F}$, and $\mathrm{G}$ which correspond to the JLab TH2, CESR analyzer magnet, and Cornell Energy Recovery Linac (ERL) CESR region magnets, respectively. Wakefields for the first two are plotted in Fig. 14, showing good agreement between the A\&Y code and Bmad. The free space steady-state wakefield Eq. (47) and results from ELEGANT are plotted for reference.

The principal detrimental effects of the CSR wake in an accelerator are energy loss and increase in energy spread of a bunch. The transverse bunch distribution can also be affected and is mostly influenced by increases of the energy spread which, through dispersive orbits, couples to transverse motion. To visualize CSR-driven energy loss and energy spread in a single magnet, we plot in Fig. 15
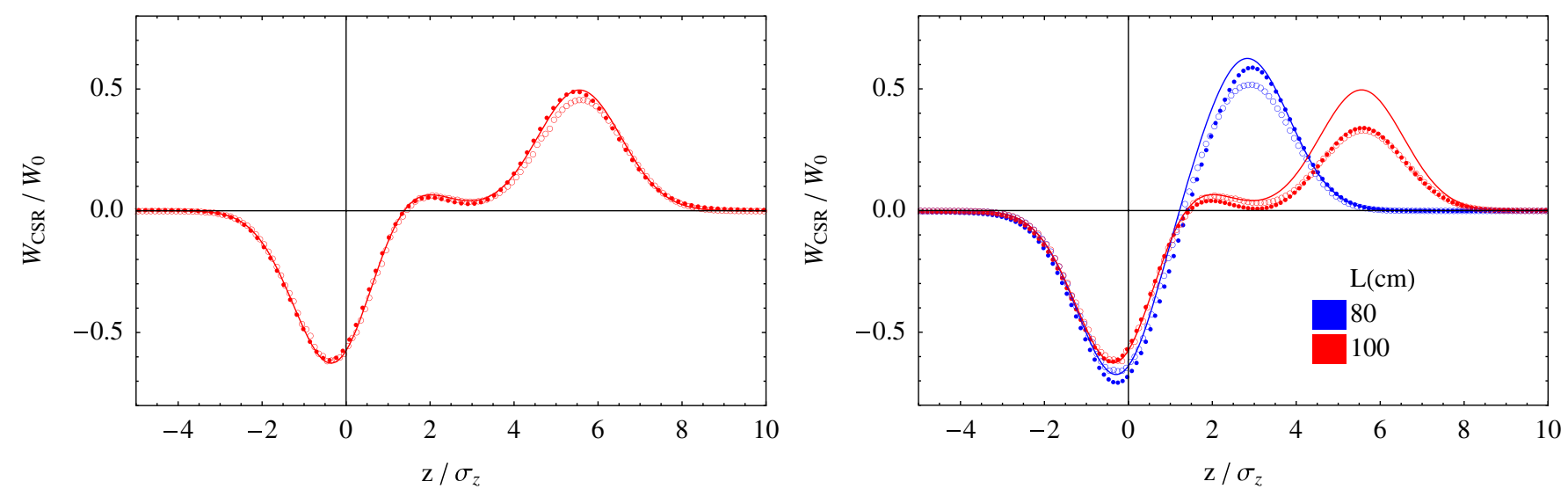

FIG. 9. (Color) Left: The transient case using parameter set B with a relatively large chamber. Right: Set C with a smaller chamber (right). A\&Y code (dots), and Bmad (circles), are generally in good agreement. The CSR-wake formula Eq. (47) (lines), which does not take into account shielding, agrees in the former case but shows differences in the latter. 

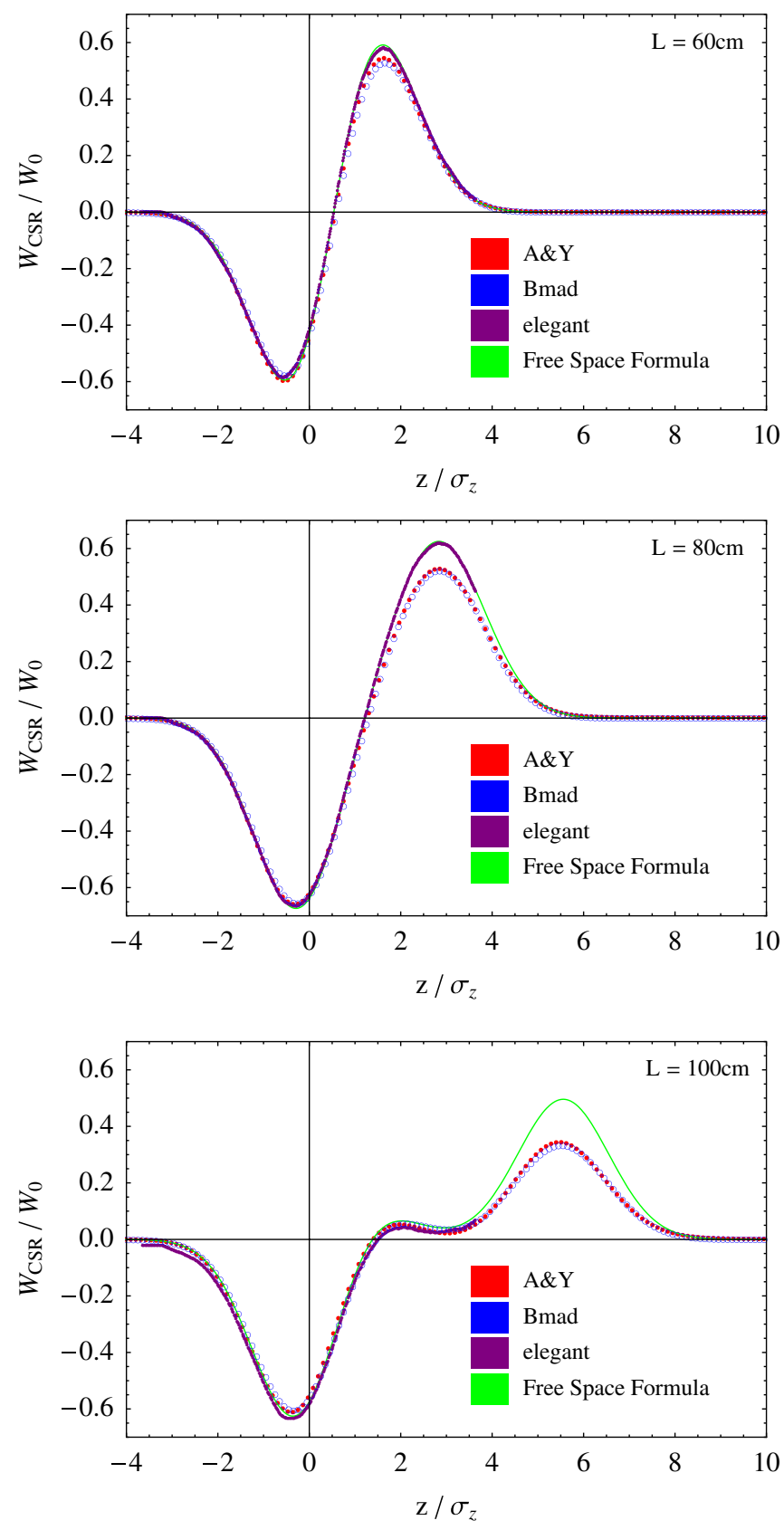

FIG. 10. (Color) Length dependence for the transient cases using the wide chamber in parameter set $\mathrm{D}$ with magnet lengths of $60 \mathrm{~cm}$ (top), $80 \mathrm{~cm}$ (middle), and $100 \mathrm{~cm}$ (bottom). The codes deviate from the CSR-wake formula of Eq. (47) for longer magnets, but Bmad (circles) agrees with A\&Y.

the average and standard deviation of the CSR wake across the bunch distribution as a function of distance into the magnet. This is done using parameter set $\mathrm{G}$, where we examine how well one can ignore the chamber width, as done in the Bmad calculation. One sees that, in this shielded case, the chamber width $w$ in the A\&Y code begins to change the wakefield when it is comparable or less than $4 \mathrm{~cm}$, the height of the chamber. This effect is

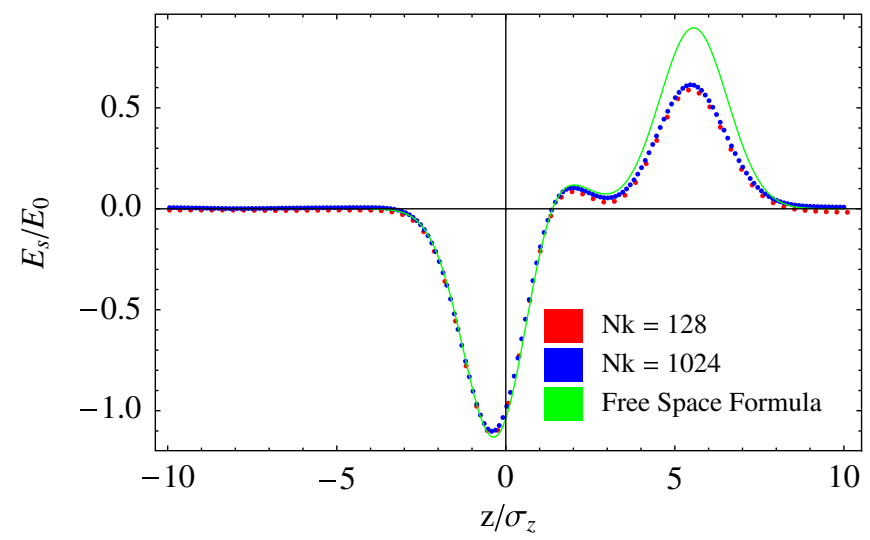

FIG. 11. (Color) Using parameter set D, varying the number of Fourier coefficients (Nk) used in the A\&Y calculation does not change the numerical solution of the Maxwell equations, and therefore does not account for deviations in the presented comparison of codes.

heuristically explained in the Appendix. It again shows that ignoring the chamber width, as in Bmad, is a reasonable approximation when the chamber width is significantly greater than its height.

It is apparent that shielding by a vacuum chamber reduces the power emitted by CSR very effectively. It does not reduce the energy spread nearly as much, and is therefore not as effective for preserving bunch properties as one might have concluded from the reduced radiation power. Interestingly, the $4 \mathrm{~cm}$ wide chamber even produces a larger standard deviation of $W_{\mathrm{CSR}}$ than wider chambers.

\section{Exit wake}

The method in this paper can correctly account for the CSR wake in a drift section following a magnet. Using parameter set A, Fig. 16 shows this wake as a function of the distance $d$ from the end of the magnet in free space and with shielding. The free space case shows excellent agreement between Bmad and the CSR-wake formula Eq. (48).

To show that Bmad correctly computes exit wakes with shielding, we compare Bmad with a numerical solution using retarded fields. In order to avoid the complication of computing retarded times and positions of the bunch distribution and its image charges, we do not use LiénardWiechert fields, but rather Jefimenko's equations [9]. In general, the electric field due to a one-dimensional charge density $\rho(s, t)$ and current density $\mathbf{J}(s, t)$ at a position $s$, time $t$, and height $h$ is

$$
\begin{aligned}
\mathbf{E}(s, t ; h)= & \frac{1}{4 \pi \epsilon_{0}} \int d s^{\prime}\left\{\frac{\mathbf{L}}{L^{3}}\left[\rho\left(s^{\prime}, t^{\prime}\right)\right]+\frac{\mathbf{L}}{c L^{2}}\left[\frac{\partial \rho\left(s^{\prime}, t^{\prime}\right)}{\partial t^{\prime}}\right]\right. \\
& \left.-\frac{1}{c^{2} L}\left[\frac{\partial \mathbf{J}\left(s^{\prime}, t^{\prime}\right)}{\partial t^{\prime}}\right]\right\},
\end{aligned}
$$

where $\mathbf{L}$ is the vector from position $s^{\prime}$ to position $s$ at height $h, L$ is its magnitude, and the brackets [] are 

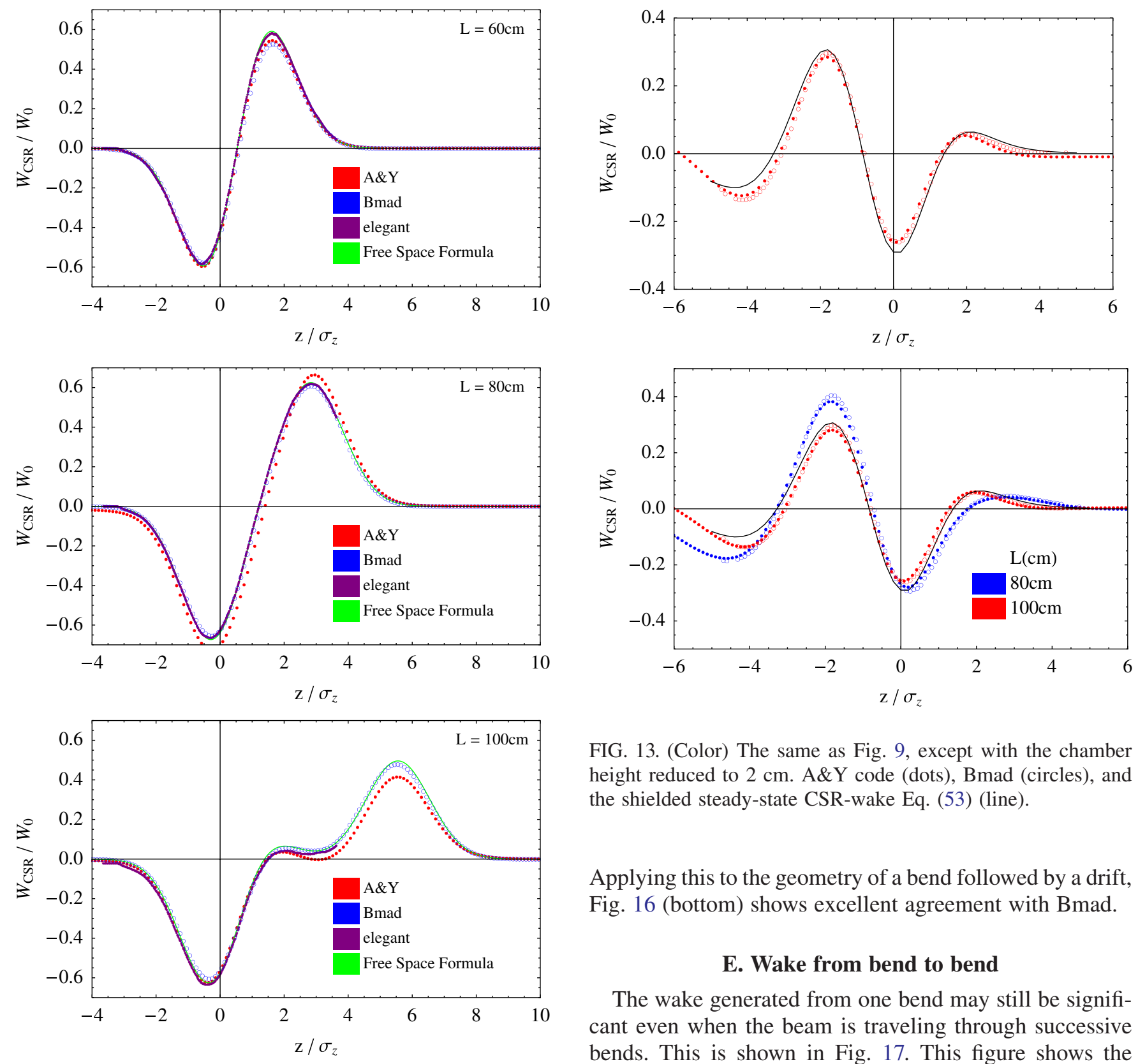

FIG. 13. (Color) The same as Fig. 9, except with the chamber height reduced to $2 \mathrm{~cm}$. A\&Y code (dots), Bmad (circles), and the shielded steady-state CSR-wake Eq. (53) (line).

Applying this to the geometry of a bend followed by a drift, Fig. 16 (bottom) shows excellent agreement with Bmad.

\section{E. Wake from bend to bend}

The wake generated from one bend may still be significant even when the beam is traveling through successive bends. This is shown in Fig. 17. This figure shows the wake, without shielding, in the second bend of a benddrift-bend configuration using the parameters from set $\mathrm{B}$ of Table I and with a $1 \mathrm{~m}$ drift between the bends.

Shown in the top graph of Fig. 17 is the wake at $1 \mathrm{~cm}$ into the bend as calculated by Bmad and ELEGANT. For reference, a third curve has been added that shows the wake, as calculated from Bmad, for a drift-bend configuration. The code of $A \& Y$ could not be used since it does not handle a bend-drift-bend configuration. ELEGANT does not take into account the wake from one drift into another so both the Bmad bend-drift and ELEGANT simulations show a negligible wake. On the other hand, the Bmad bend-drift-bend simulation shows a substantial wake.

Shown in the bottom graph of Fig. 17 is the wake at $100 \mathrm{~cm}$ into the bend as calculated by Bmad and ELEGANT. Again, for reference, a third curve has been added that 

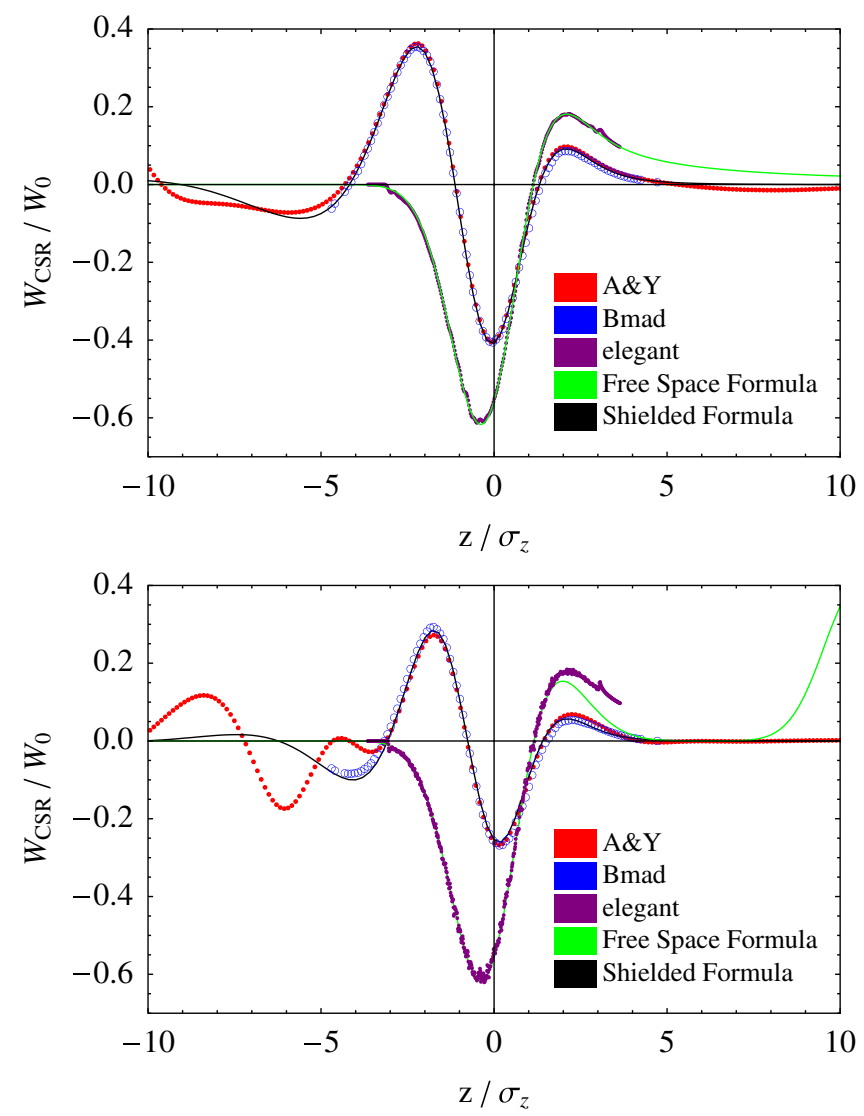

FIG. 14. (Color) Realistic magnets: Parameter set E (JLab TH2 magnet) line (top), set F (CESR analyzer magnet) (bottom). Bmad agrees with the CSR-wake formula Eq. (53) better than the other codes at the bunch tail.

shows the wake, as calculated from Bmad, for a drift-bend configuration. Here, the wakefield for $z / \sigma_{z} \lesssim 2$ is the same with and without the initial bend indicating that the wakefield in this region is principally formed in the second bend. However, for $z / \sigma_{z} \gtrsim 2$ the wake from the first bend is clearly important.

\section{F. Coherent energy loss}

In general, for $N$ particles traveling on the same curve at different phases $\phi_{n}$, the $N$ particle power spectrum is

$$
\begin{aligned}
\frac{d P^{(N)}}{d \omega}= & \left|\sum_{n=1}^{N} e^{i \phi_{n}}\right| \frac{d P^{(1)}}{d \omega}=\left(N+\sum_{m \neq n} e^{i\left(\phi_{m}-\phi_{n}\right)}\right) \frac{d P^{(1)}}{d \omega} \\
\simeq & N \frac{d P^{(1)}}{d \omega}+N(N-1)\left|\int d z \lambda(z) \exp \left(i \frac{\omega z}{\beta c}\right)\right|^{2} \\
& \times \frac{d P^{(1)}}{d \omega},
\end{aligned}
$$

where $d P^{(1)} / d \omega$ is the single particle power spectrum, and $\lambda(z)$ is the longitudinal particle distribution. The first term

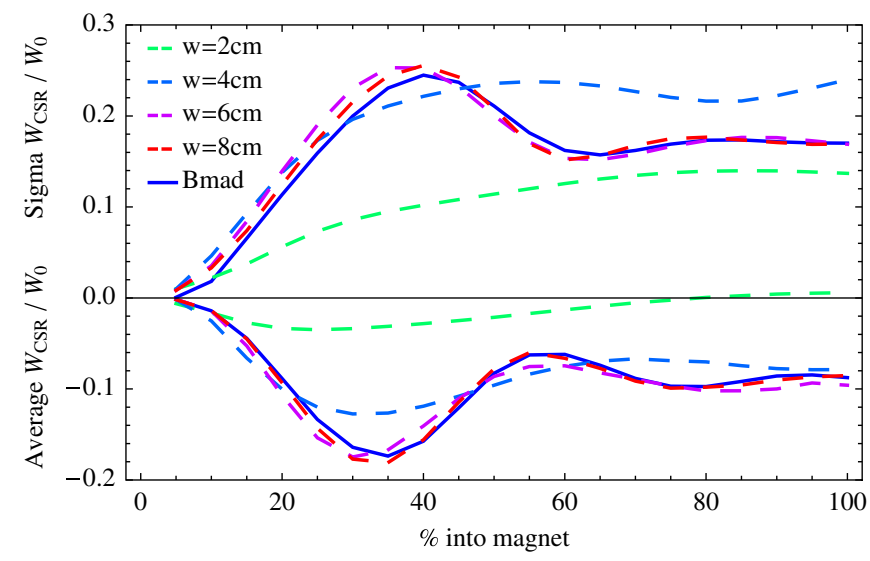

FIG. 15. (Color) Average and standard deviation of the CSR wake across the bunch distribution for parameter set $G$ (the Cornell ERL's CE magnet) for various chamber widths using the A\&Y code, compared to Bmad, which has an infinite chamber width.

in Eq. (59) is the incoherent power spectrum, while the second is the coherent power spectrum. In the presence of conducting parallel plates, the single particle power spec-
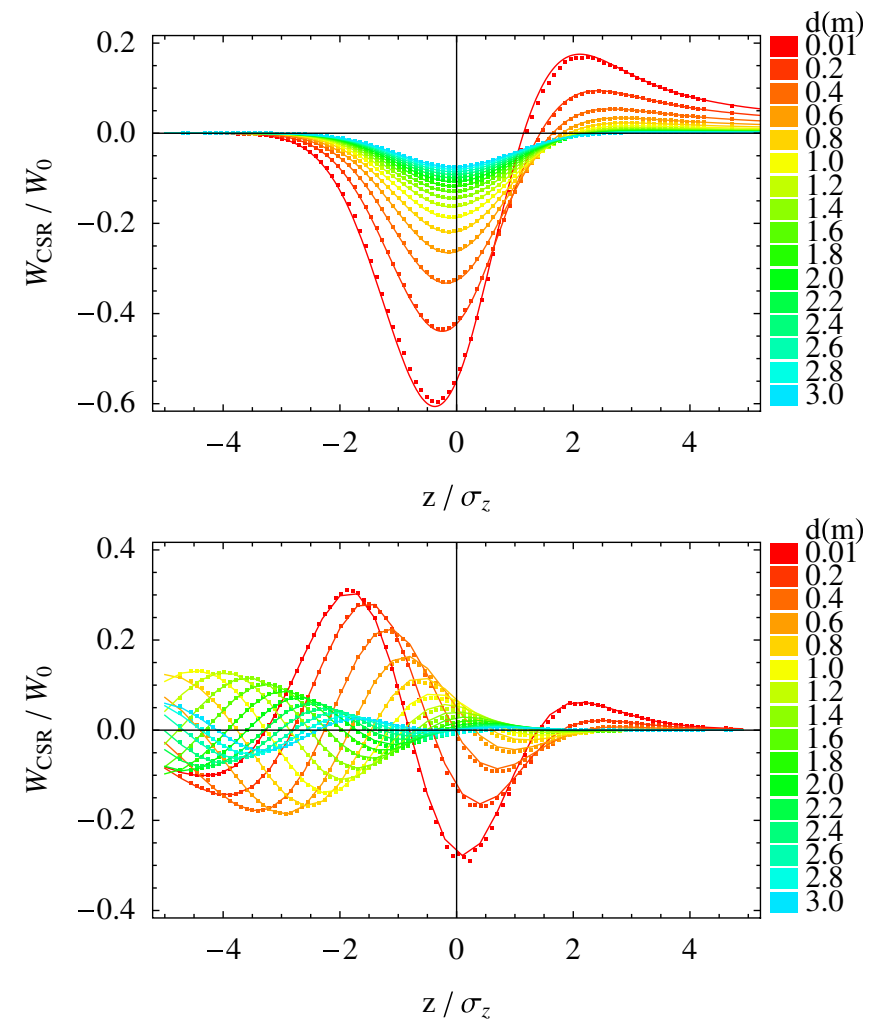

FIG. 16. (Color) Exit wakefield as a function of the length $d$ from the end of the magnet for free space (top) and with shielding (bottom). Bmad (dots) shows excellent agreement with CSR-wake formula Eq. (48) (top lines) in the free space case, and with numerical integration over image bunches using Eq. ((47) (bottom lines). Parameters set A is used. 

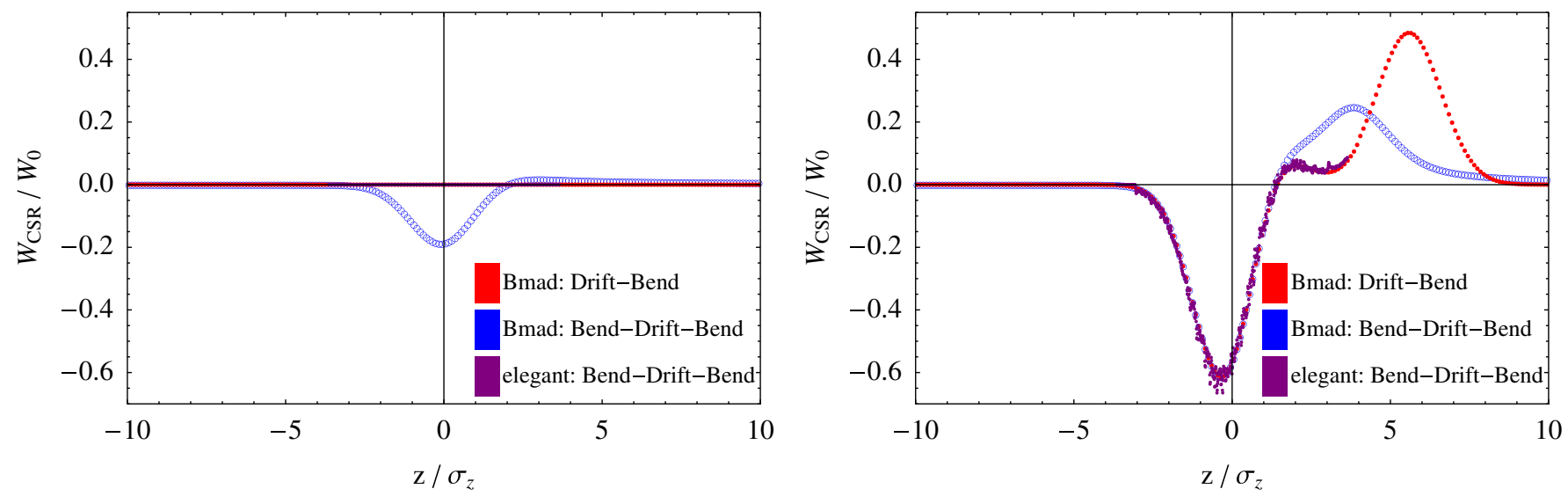

FIG. 17. (Color) The wake in the second bend of a bend-drift-bend configuration as calculated by Bmad and ELEGANT. For reference, a third curve has been added that shows the wake, as calculated from Bmad, for a drift-bend configuration. The parameters from set B of Table I and with a $1 \mathrm{~m}$ drift between the bends was used. Top: The wake after $1 \mathrm{~cm}$ into the bend. Bottom: The wake after $100 \mathrm{~cm}$ into the bend.

trum is given in Eq. (47) of [1]. Using this, Eq. (59) can be integrated numerically.

As a test of Bmad, Fig. 18 shows the total coherent energy lost for various particle energies and chamber heights as calculated using Bmad and as calculated by
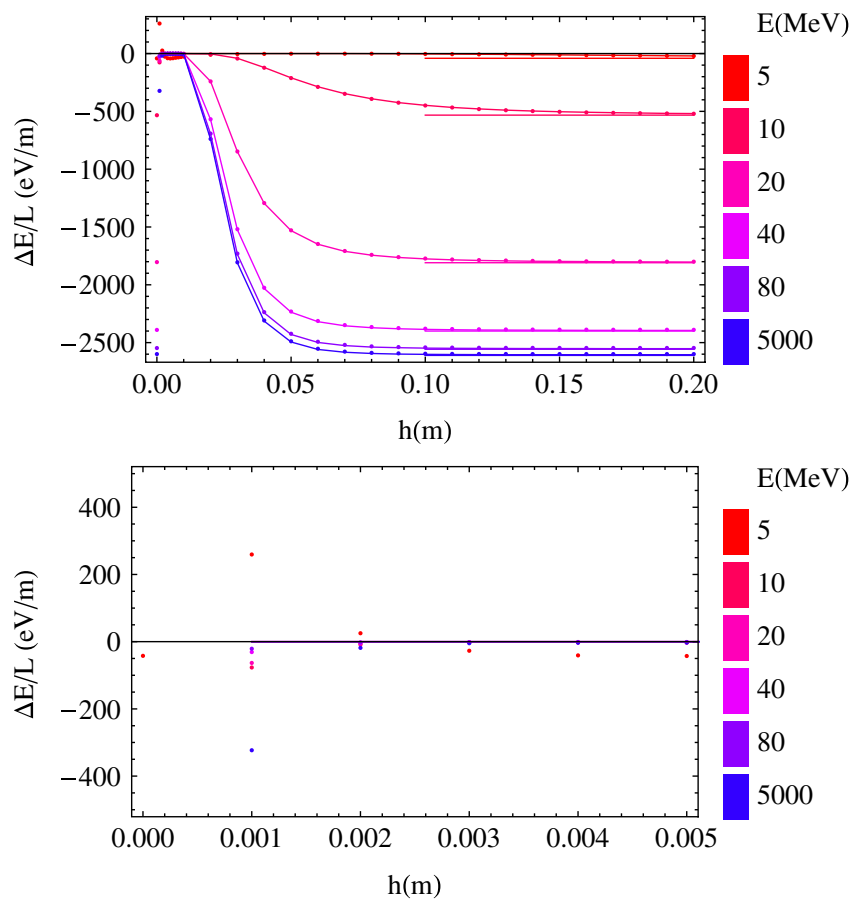

FIG. 18. (Color) Average energy losses versus shielding height for various energies. The bottom plot is the same as the top highlighting the smaller heights. Bmad (dots) agrees well with numerical integration of Eq. (59) (curved lines) using the shielded power spectrum of [1] down to $2 \mathrm{~mm}$. Steady-state losses computed using Eq. (60) are indicated as horizontal lines. Parameters set A is used, with the number of image layers $N_{i}=$ 64. integration of the power spectrum. The figure shows excellent agreement for energies down to $5 \mathrm{MeV}$ and chamber heights down to $2 \mathrm{~mm}$. At smaller heights, the number of image layers used in the simulation $\left(N_{i}=64\right.$ here $)$ is not sufficient to correctly model the CSR.

In the absence of shielding plates, Eq. (59) can be integrated exactly for a Gaussian distribution using the well-known free space single particle power spectrum (see Ref. [14]). For a bunch length of $\sigma_{z}$, the total power lost by $N$ particles is

$$
P^{(N)}=P^{(1)} N+P^{(1)} N(N-1) T\left(\frac{3 \sigma_{z} \gamma^{3}}{2 R \beta}\right)
$$

where

$$
P^{(1)} \equiv \frac{2}{3} r_{c} m c^{3} \frac{\beta^{4} \gamma^{4}}{R^{2}}
$$

is the power lost by a single particle, and

$$
T(a) \equiv \frac{9}{32 \sqrt{\pi} a^{3}} \exp \left(\frac{1}{8 a^{2}}\right) K_{5 / 6}\left(\frac{1}{8 a^{2}}\right)-\frac{9}{16 a^{2}} .
$$

This result agrees well with Bmad in Fig. 18. The function $T(a)$ can be expanded asymptotically, giving the leading order coherent energy change in a length $L$ as

$$
\Delta E^{(N)} \simeq-N^{2} r_{c} m c^{2} \frac{\Gamma\left(\frac{5}{6}\right)}{6^{1 / 3} \sqrt{\pi}} \frac{L}{\left(R^{2} \sigma_{z}^{4}\right)^{1 / 3}} .
$$

This is proportional to $W_{0}$ in Eq. (55), which is the reason for using such a scale factor. 


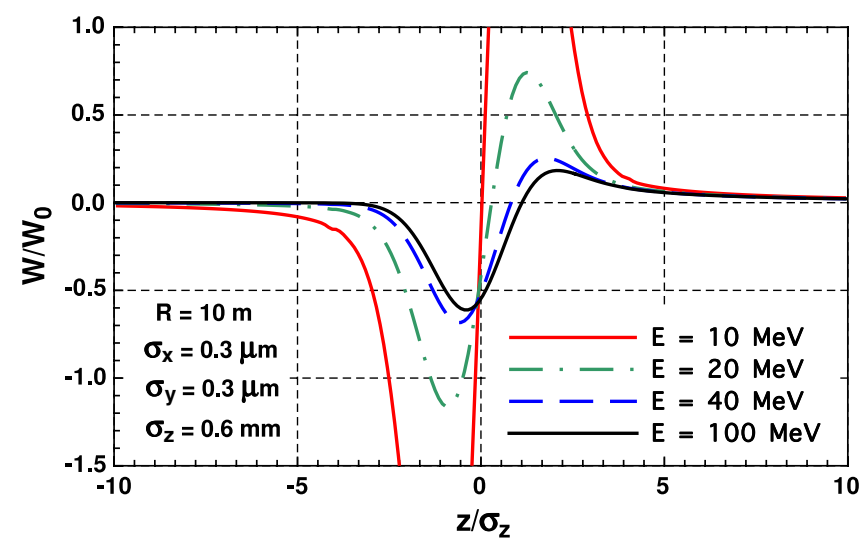

FIG. 19. (Color) The full (CSR + SC) steady-state kick as a function of longitudinal position for four different energies between 10 and $100 \mathrm{MeV}$.

\section{BEAM ENERGY DEPENDENCE OF THE ENERGY LOSS}

In the ultrarelativistic limit, the energy loss due to CSR is independent of the beam energy [for example, see Eq. (21)] and, as shown in Eq. (37), the SC loss is negligible. The situation is quite different at lower energies. This is shown by Fig. 19 which shows the full (CSR + SC) steady-state longitudinal kick as a function of longitudinal position at four different beam energies between 10 and $100 \mathrm{MeV}$ for a Gaussian bunch without shielding. The parameters chosen in this simulation are appropriate for the Cornell ERL merger. As can be seen from the figure, there is an increase in energy loss for $z<0$ and increase in energy gain for $z>0$ as the beam energy is lowered.

That this increase in energy variation with decreasing beam energy is due to the SC kick component is illustrated in Figs. 20 and 21 which show the steady-state kick with and without the SC component at energies of 20 and $40 \mathrm{MeV}$. In fact, the energy variation due to the CSR component alone decreases with decreasing beam energy.

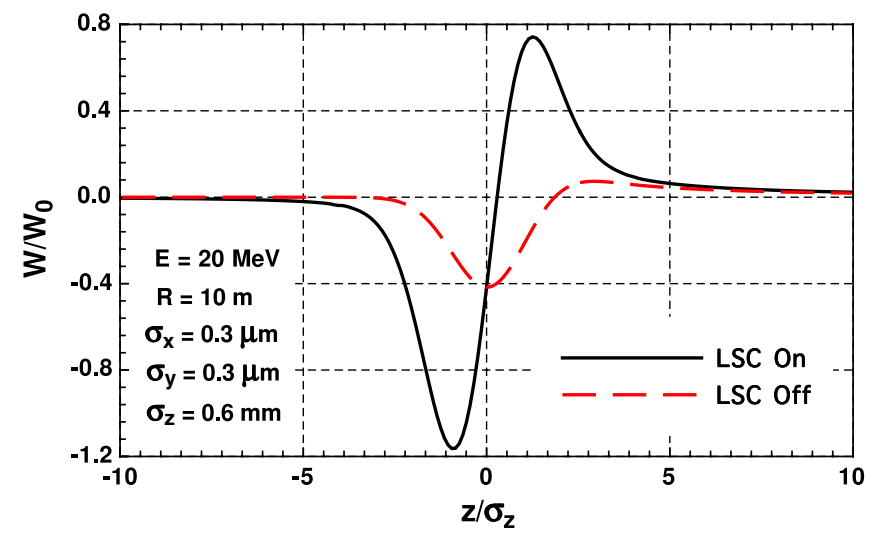

FIG. 20. (Color) The steady-state kick due to CSR alone and the combined CSR and SC kick for a $20 \mathrm{MeV}$ beam.

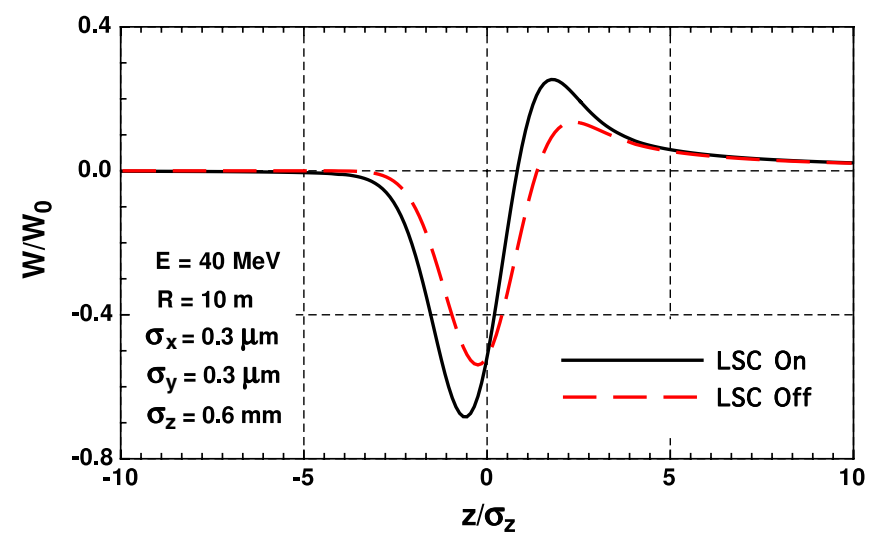

FIG. 21. (Color) The steady-state kick due to CSR alone and the combined CSR and SC kick for a $40 \mathrm{MeV}$ beam.

At $40 \mathrm{MeV}$ the SC component is significant but not dominant. At $20 \mathrm{MeV}$ the situation is reversed. In this energy range the variable $M$ from Eq. (39) is 2.7 to a good approximation, and which according to Eq. (37) gives a crossover energy of $69 \mathrm{MeV}$. That the simulation indicates a lower energy for the crossover can be explained by noting that the CSR calculation assumes that all particles are on axis and it does not take into account any falloff in CSR kick with increasing transverse kick.

\section{CONCLUSION}

A formalism for calculating the longitudinal kick due to coherent synchrotron radiation originally due to Saldin, Schneidmiller, and Yurkov has been implemented in Bmad along with a heuristic formula for the longitudinal space charge kick. The space charge kick is only significant at lower particle energies where the neglect of any transverse forces in the formalism may make simulations inaccurate. However, the formulas developed allow one to determine if the ultrarelativistic approximation is warranted.

We show that the longitudinal wakefield compares well with the code of Agoh and Yokoya [12] and the CSR-wake formula of Warnock [2] for the steady state, with and without CSR shielding by parallel plates. In the transient case where the A\&Y code often does not follow the CSRwake formula of Eq. (47) exactly, Bmad does agree well with that of Agoh and Yokoya. This agreement is comforting but not surprising because related approximations underlie each of the approaches. Comparison to the CSRwake formula may indicate the level of accuracy of the Bmad approach. A detailed analysis of the accuracy is presented in [14].

\section{ACKNOWLEDGMENTS}

The authors would like to acknowledge help from Tsukasa Miyajima and Ivan V. Bazarov. We also thank Michael Borland for useful discussions. This work has 


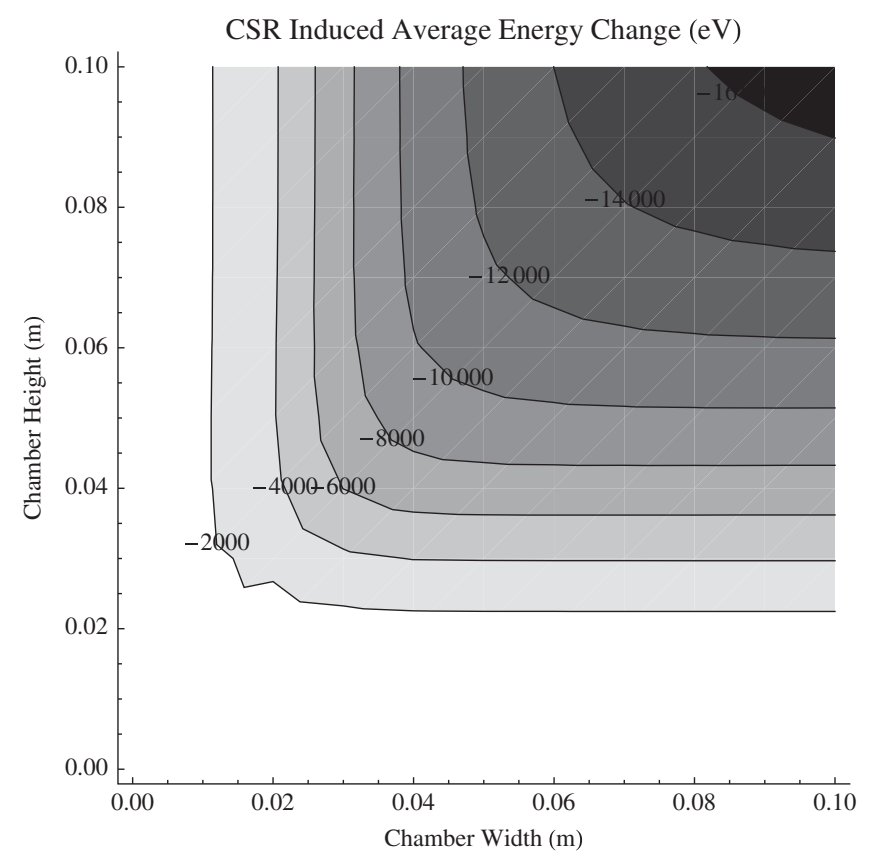

FIG. 22. Numerical results using the A\&Y code with varying dimensions of a rectangular chamber. A $1 \mathrm{~m}, 7.5^{\circ}$ bend with a $2 \mathrm{ps}$ long bunch of $0.82 \mathrm{nC}$ charge was used. Contours represent the energy change induced due to the CSR wakefield in $2000 \mathrm{eV}$ increments. The shielding effect is thus primarily due to the smaller of the two beam-pipe dimensions.

been supported by NSF Cooperative Agreement No. PHY0202078.

\section{APPENDIX A: HEURISTIC SHIELDING ARGUMENT}

Initially, it might seem surprising that a vacuum chamber whose width is on the order of centimeters can have a shielding effect on synchrotron radiation whose wavelength is much smaller. We therefore add a heuristic explanation here.

Starting with Schwinger [1], shielding by a vacuum chamber has often been considered by studying infinite horizontal, conducting plates. But the following heuristic argument indicates why it is both the vertical and the horizontal boundary that determines shielding of CSR.

A highly relativistic particle emits synchrotron radiation within a narrow cone. For the radiation's component of wavelength $\lambda$, the opening angle of this cone is approximately $\Delta \theta=\left(\frac{\lambda}{R}\right)^{1 / 3}$ in the horizontal and vertical. The opening angle in the vertical determines the vertical width of the radiation load on the vacuum chamber wall.

The radiation field builds up within a radiation buildup time of $\Delta t=\frac{R}{c}\left(\frac{\lambda}{R}\right)^{1 / 3}$. During this time, the radiation fields produced by the electron coherently add up to form the full radiation power. If the radiation does not interfere with an obstacle, for example, the vacuum pipe, within this time, the electron loses as much energy as it would without any vacuum pipe.

The width and height of the radiation cone that builds up during the radiation buildup time is therefore given by $w_{r} \approx h_{r} \approx c \Delta t \Delta \theta=R\left(\frac{\lambda}{R}\right)^{2 / 3}$. Vacuum chambers that have smaller dimensions interfere with the radiation process and shield the part of radiation for which

$$
\lambda \gtrsim \min \left[w \sqrt{\frac{w}{R}}, h \sqrt{\frac{h}{R}}\right] .
$$

Wavelengths are therefore shielded when they are above a length that is much smaller than the chamber dimensions.

While we have used a very approximate heuristic argument, Fig. 22, which shows the CSR induced average energy change as a function of chamber height and width as computed by the A\&Y code, indeed shows that both dimensions can lead to shielding. The figure also shows that, in the first approximation, only the smaller of the two dimensions is relevant.

[1] J. Schwinger, A Quantum Legacy: Seminal Papers of Julian Schwinger, edited by Kimball A. Milton (World Scientific, Singapore, 2000), p. 307.

[2] R. Warnock, Report No. SLAC-PUB-5375, 1990.

[3] J. B. Murphy, "An Introduction to Coherent Synchrotron Radiation in Storage Rings," ICFA Beam Dynamics Newsletter, No. 35 (2004).

[4] E. L. Saldin, E. A. Schneidmiller, and M. V. Yurkov, Nucl. Instrum. Methods Phys. Res., Sect. A 417, 158 (1998).

[5] Y.S. Derbenev, J. Rossbach, E. L. Saldin, and V.D. Shiltsev, TESLA FEL-Report No. 1995-05, 1995.

[6] E. L. Saldin, E. A. Schneidmiller, and M. V. Yurkov, Nucl. Instrum. Methods Phys. Res., Sect. A 398, 373 (1997).

[7] D. Sagan, Nucl. Instrum. Methods Phys. Res., Sect. A 558, 356 (2006).

[8] G. Bassi et al., Nucl. Instrum. Methods Phys. Res., Sect. A 557, 189 (2006).

[9] J.D. Jackson, Classical Electrodynamics (Wiley, New York, 1999), p. 678, 3rd ed.

[10] P. Emma and G. V. Stupakov, Proceedings of the 8th European Particle Accelerator Conference, Paris, 2002 (EPS-IGA and CERN, Geneva, 2002), p. 1479.

[11] W. Press, B. Flannery, S. Teukolsky, and W. Wetterling, Numerical Recipes in Fortran, The Art of Scientific Computing (Cambridge University Press, New York, 1992), 2nd ed.

[12] T. Agoh and K. Yokoya, Phys. Rev. ST Accel. Beams 7, 054403 (2004).

[13] M. Borland, Phys. Rev. ST Accel. Beams 4, 070701 (2001).

[14] C. E. Mayes and G. H. Hoffstaetter, Phys. Rev. ST Accel. Beams 12, 024401 (2009). 